\title{
On existence of solutions of a impulsive stochastic partial functional integro-differential equation with the measure of noncompactness
}

Zuomao Yan ${ }^{*}$ and Xiumei Jia

"Correspondence:

yanzuomao@163.com

Department of Mathematics, Hexi

University, Zhangye, Gansu 734000,

P.R. China

\section{照 Springer}

\begin{abstract}
In this article, we study the existence of mild solutions for a new class of impulsive stochastic partial neutral functional integro-differential equations with infinite delay and non-instantaneous impulses in separable Hilbert spaces. The new results are obtained by using the Hausdorff measure of noncompactness, and the theory of analytic resolvent operators and fractional power of closed operators with the fixed point theorems. An example is also given to illustrate the obtained theory.
\end{abstract}

MSC: $34 \mathrm{~A} 37 ; 34 \mathrm{~A} 60 ; 34 \mathrm{~K} 40 ; 60 \mathrm{H} 15$

Keywords: impulsive stochastic partial neutral functional integro-differential equations; Hausdorff measure of noncompactness; infinite delay; analytic resolvent operators; fixed point theorem

\section{Introduction}

The study of impulsive functional differential and integro-differential systems is linked to their utility in simulating processes and phenomena subject to short-time perturbations during their evolution. The perturbations are performed discretely and their duration is negligible in comparison with the total duration of the processes and phenomena. Now impulsive partial neutral functional differential and integro-differential systems have become an important object of investigation in recent years stimulated by their numerous applications to problems arising in mechanics, electrical engineering, medicine, biology, ecology, etc. With regard to this matter, we refer the reader to [1-4] and the references therein.

Stochastic differential equations have attracted great interest due to their applications in characterizing many problems in physics, biology, mechanics, and so on; see [5]. There are many publications in the qualitative properties of solutions for these equations (see [6-9] and the references therein). As the generalization of classic impulsive differential equations, impulsive partial stochastic differential and integro-differential equations have been extensively studied in Hilbert spaces. For example, Sakthivel and Luo [10] studied the existence and asymptotic stability in the $p$ th moment of mild solutions to impulsive stochastic partial differential equations with and without infinite delays through

(c) 2016 Yan and Jia. This article is distributed under the terms of the Creative Commons Attribution 4.0 International License (http://creativecommons.org/licenses/by/4.0/), which permits unrestricted use, distribution, and reproduction in any medium, provided you give appropriate credit to the original author(s) and the source, provide a link to the Creative Commons license, and indicate if changes were made. 
fixed point theory. Anguraj and Vinodkumar [11] investigated the existence, uniqueness, and stability of mild solutions of impulsive stochastic semilinear neutral functional differential equations without a Lipschitz condition and with a Lipschitz condition. Hu and Ren [12] proved the existence of mild solutions for a class of impulsive neutral stochastic functional integro-differential equations with infinite delays. Lin et al. [13] discussed the existence of mild solutions for a class of neutral impulsive stochastic integro-differential equations with infinite delays and analytic resolvent operators. In [14], the authors studied the existence of mild solutions for a class of impulsive partial neutral stochastic integrodifferential equation with state-dependent delay. Further, Sakthivel et al. [15] were concerned with the existence of mild solutions for the fractional impulsive stochastic differential equations with infinite delay in Hilbert spaces. Yan and Zhang [16] obtained the existence and asymptotic stability of solutions to fractional impulsive neutral stochastic partial integro-differential equations with state-dependent delay. Balasubramaniam et al. [17] discussed the local and global existence of mild solutions studied for impulsive fractional semilinear stochastic differential equations with a nonlocal condition in a Hilbert space. Ren et al. [18] proved the existence and uniqueness of the mild solution for impulsive neutral stochastic functional integro-differential equations with infinite delay driven by $\mathrm{fBm}$.

In fact, for these abstract impulsive stochastic partial differential and integro-differential equations one considers basically problems for which the impulses are abrupt and instantaneous. However, many impulsive systems arising from realistic models can be described as partial differential equations and integro-differential with non-instantaneous impulses. The significance of the study of these equations lies in its diverse fields of applications such as in the theory of stage by stage rocket combustion, maintaining hemodynamical equilibrium (see [2]). Recently, Hernández and O'Regan [19] introduced a new class of first order abstract impulsive differential equations for which the impulses are not instantaneous. In the model, the impulses start abruptly at the points $t_{i}$ and their action continues on a finite time interval $\left[t_{i}, s_{i}\right]$. This is a situation with an impulsive action which starts abruptly and stays active on a finite time interval. Pierri et al. [20] studied the existence of solutions for a class of first order semilinear abstract impulsive differential equations with non-instantaneous impulses by using the theory of analytic semigroup and fractional power of closed operators. Gautam and Dabas [21] established the existence, uniqueness, and continuous dependence results of mild solution fractional functional integro-differential equations with non-instantaneous impulse. Yu and Wang [22] discussed the existence of mild solutions for periodic boundary value problems with noninstantaneous impulse on Banach spaces. Fu et al. [23] were concerned with the existence of mild solutions for Cauchy and nonlocal problems of impulsive fractional evolution equations for which the impulses are not instantaneous. Many control systems arising from realistic models can be described as stochastic systems with non-instantaneous impulses. So it is natural to extend the concept of the existence to dynamical systems represented by these impulsive systems. Yan and $\mathrm{Lu}$ [24] discussed a class of fractional impulsive partial stochastic integro-differential equations with non-instantaneous impulses in Hilbert spaces under the Lipschitz conditions. In this paper we consider the existence of a new class of impulsive stochastic partial neutral functional integro-differential equations with infinite delay and non-instantaneous impulses of the form in Hilbert spaces of the 
form

$$
\begin{aligned}
& d\left[x(t)-G\left(t, x_{t}\right)\right]=A\left[x(t)+\int_{0}^{t} h(t-s) x(s) d s\right] d t+f\left(t, x_{t}\right) d t+F\left(t, x_{t}\right) d w(t), \\
& \quad t \in\left(s_{i}, t_{i+1}\right], i=0,1, \ldots, N, \\
& x_{0}=\varphi \in \mathcal{B}, \\
& x(t)=g_{i}\left(t, x_{t}\right), \quad t \in\left(t_{i}, s_{i}\right], i=1, \ldots, N,
\end{aligned}
$$

where the state $x(\cdot)$ takes values in a separable real Hilbert space $H$ with inner product $(\cdot, \cdot)$ and norm $\|\cdot\|$, and $A$ is the infinitesimal generator of an analytic resolvent operator $\{R(t)\}_{t \geq 0}$ in $H$. The history $x_{t}:(-\infty, 0] \rightarrow H, x_{t}(\theta)=x(t+\theta)$, belongs to some abstract phase space $\mathcal{B}$ defined axiomatically in Section 2. Let $K$ be another separable Hilbert space with inner product $(\cdot, \cdot)_{K}$ and norm $\|\cdot\|_{K}$. Suppose $\{w(t): t \geq 0\}$ is a given $K$-valued Brownian motion or Wiener process with a finite trace nuclear covariance operator $Q>0$ defined on a complete probability space $(\Omega, \mathcal{F}, P)$ equipped with a normal filtration $\left\{\mathcal{F}_{t}\right\}_{t \geq 0}$, which is generated by the Wiener process $w . h(t), t \in[0, b]$, and is a bounded linear operator, and let $0=t_{0}=s_{0}<t_{1} \leq s_{1} \leq t_{2}<\cdots<t_{N-1} \leq s_{N} \leq t_{N} \leq t_{N+1}=b$, be prefixed numbers, $g_{i} \in C\left(\left(t_{i}, s_{i}\right] \times \mathcal{B} ; H\right)$ for all $i=1, \ldots, N$, and $G, f, F$ are given functions to be specified later. The initial data $\{\varphi(t):-\infty<t \leq 0\}$ is an $\mathcal{F}_{0}$-adapted, $\mathcal{B}$-valued random variable independent of the Wiener process $w$ with finite second moment.

The authors [19-24] supposed that the impulsive systems with the compactness assumption on associated operators and the nonlinear function is a Lipschitz function or is completely continuous. However, the above conditions are stronger restrictions, which are not satisfied usually in many practical problems $[25,26]$. We can use a measure of noncompactness to remove the assumptions for the compactness of the operator and Lipschitz continuity of the nonlinear item. To the best of our knowledge, there are no relevant reports on the impulsive stochastic differential equations with non-instantaneous impulses via the techniques of the measure of noncompactness. This is one of our motivations.

In this article, we shall study the existence of mild solutions of (1.1)-(1.3) by using the Hausdorff measure of noncompactness, the Darbo fixed point theorem, Darbo-Sadovskii fixed point theorem with the theory of analytic resolvent operators and fractional power of closed operators. We do not assume $\{R(t), t \geq 0\}$ is a compact semigroup, and instead we assume that $f, F$ satisfy a compactness condition involving the Hausdorff measure of noncompactness. Thus the compactness of $R(t)$ or $f, F$ and the Lipschitz condition of $f, F$ are the special cases of our conditions. The known results appearing in [19-24] are generalized to the impulsive stochastic systems settings and the case of infinite delay without the assumptions of compactness. The nonlinear integro-differential equation with resolvent operators serves as an abstract formulation of partial integrodifferential equations which arises in many physical phenomena [27-29]. The resolvent operator is similar to the semigroup operator for abstract differential equations in Banach spaces. However, the resolvent operator does not satisfy the semigroup properties. Our main results can be seen as a generalization of the work in [12-14] and the above mentioned equations with non-instantaneous impulses. Moreover, motivated by the results, we see the direct issues which require further study. For instance, we 
will investigate the qualitative properties of solutions for a new class of impulsive partial stochastic differential systems with non-instantaneous impulses by using resolvent operators, such as controllability, approximate controllability, optimal control, and stability. Also, we will address the study of the existence and qualitative analysis of solutions of impulsive partial stochastic differential inclusions with non-instantaneous impulses.

The rest of this paper is organized as follows. In Section 2, we introduce some notations and necessary preliminaries. In Section 3, we give our main results. In Section 4, an example is given to illustrate our results. Finally, concluding remarks are given in Section 5.

\section{Preliminaries}

Let $(\Omega, \mathcal{F}, P)$ be a complete probability space equipped with a normal filtration $\mathcal{F}_{t}, t \in$ $[0, b]$. Let $H$ and $K$ be separable Hilbert spaces, $L(K, H)$ be the space of linear operators mapping $K$ into $H$, and $L(K, H)$ be the space of bounded linear operators mapping $K$ into $H$ equipped with the usual norm $\|\cdot\|_{H}, L(H)$ denote the Hilbert space of bounded linear operators from $H$ to $H$, and $w$ be a $Q$-Weiner process on $\left(\Omega, \mathcal{F}_{b}, P\right)$ with the covariance operator $Q$ such that $\operatorname{Tr} Q<\infty$. We assume that there exists a complete orthonormal system $\left\{e_{n}\right\}_{n=1}^{\infty}$ in $K$, a bounded sequence of nonnegative real numbers $\left\{\lambda_{n}\right\}_{n=1}^{\infty}$ such that $Q e_{n}=\lambda_{n} e_{n}$, and a sequence $\beta_{n}$ of independent Brownian motions such that

$$
\langle w(t), e\rangle=\sum_{n=1}^{\infty} \sqrt{\lambda_{n}}\left\langle e_{n}, e\right\rangle \beta_{n}(t), \quad e \in K, t \in[0, b]
$$

and $\mathcal{F}_{t}=\mathcal{F}_{t}^{w}$, where $\mathcal{F}_{t}^{w}$ is the $\sigma$-algebra generated by $\{w(s): 0 \leq s \leq t\}$. Let $L_{2}^{0}=$ $L_{2}\left(Q^{1 / 2} K ; H\right)$ be the space of all Hilbert-Schmidt operators from $Q^{1 / 2} K$ to $H$ with the inner product $(\psi, \tilde{\theta})_{L_{2}^{0}}=\operatorname{Tr}\left(\psi Q \tilde{\theta}^{*}\right)$.

The collection of all strongly measurable, square integrable, $H$-valued random variables, denoted by $L_{2}(\Omega, H)$, is a Banach space equipped with norm $\|x(\cdot)\|_{L_{2}}=\left(E\|x(\cdot, w)\|^{2}\right)^{\frac{1}{2}}$, where the expectation, $E$, is defined by $E x=\int_{\Omega} x(w) d P$. Let $C\left([0, b], L_{2}(\Omega, H)\right)$ be the Banach space of all continuous maps from $J$ into $L_{2}(\Omega, H)$ satisfying the condition $\sup _{0 \leq t \leq b} E\|x(t)\|^{2}<\infty$. Let $L_{2}^{0}(\Omega, H)$ denote the family of all $\mathcal{F}_{0}$-measurable, $H$-valued random variables $x(0)$. The notation, $B_{r}(x, H)$ stands for the closed ball with center at $x$ and radius $r>0$ in $H$.

In this paper, we assume that the phase space $\left(\mathcal{B},\|\cdot\|_{\mathcal{B}}\right)$ is a seminormed linear space of $\mathcal{F}_{0}$-measurable functions mapping $(-\infty, 0]$ into $H$, satisfying the following fundamental axioms due to Hale and Kato (see, e.g., [30]).

(A) If $x:(-\infty, \sigma+b] \rightarrow H, b>0$, is such that $\left.x\right|_{[\sigma, \sigma+b]} \in C([\sigma, \sigma+b], H)$ and $x_{\sigma} \in \mathcal{B}$, then for every $t \in[\sigma, \sigma+b]$ the following conditions hold:

(i) $x_{t}$ is in $\mathcal{B}$;

(ii) $\|x(t)\| \leq \tilde{H}\left\|x_{t}\right\|_{\mathcal{B}}$

(iii) $\left\|x_{t}\right\|_{\mathcal{B}} \leq K(t-\sigma) \sup \{\|x(s)\|: \sigma \leq s \leq t\}+M(t-\sigma)\left\|x_{\sigma}\right\|_{\mathcal{B}}$, where $\tilde{H}>0$ is a constant; $K, M:[0, \infty) \rightarrow[1, \infty), K$ is continuous and $M$ is locally bounded, and $\tilde{H}, K, M$ are independent of $x(\cdot)$.

(B) For the function $x(\cdot)$ in (A), the function $t \rightarrow x_{t}$ is continuous from $[\sigma, \sigma+b]$ into $\mathcal{B}$.

(C) The space $\mathcal{B}$ is complete. 
Remark 2.1 ([3]) Let $\varphi \in \mathcal{B}$ and $t \leq 0$. The notation $\varphi_{t}$ represents the function defined by $\varphi_{t}=\varphi(t+\theta)$. Consequently, if the function $x(\cdot)$ in axiom (A) is such that $x_{0}=\varphi$, then $x_{t}=\varphi_{t}$. We observe that $\varphi_{t}$ is well defined for $t<0$ since the domain of $\varphi$ is $(-\infty, 0]$.

Remark 2.2 ([3]) In retarded functional differential equations without impulses, the axioms of the abstract phase space $\mathcal{B}$ include the continuity of the function $t \rightarrow x_{t}$. Due to the impulsive effect, this property is not satisfied in impulsive delay systems and, for this reason, has been eliminated in our abstract description of $\mathcal{B}$.

The next result is a consequence of the phase space axioms.

Lemma 2.1 (Compare with [31]) Let $x:(-\infty, b] \rightarrow H$ be an $\mathcal{F}_{t}$-adapted measurable process such that the $\mathcal{F}_{0}$-adapted process $x_{0}=\varphi(t) \in L_{2}^{0}(\Omega, \mathcal{B})$ and $\left.x\right|_{[0, b]} \in \mathcal{P C}([0, b], H)$, then

$$
\left\|x_{s}\right\|_{\mathcal{B}} \leq M_{b} E\|\varphi\|_{\mathcal{B}}+K_{b} \sup _{0 \leq s \leq b} E\|x(s)\|
$$

where $K_{b}=\sup \{K(t): 0 \leq t \leq b\}, M_{b}=\sup \{M(t): 0 \leq t \leq b\}$.

We introduce the space $\mathcal{P C}$ formed by all $\mathcal{F}_{t}$-adapted measurable, $H$-valued stochastic processes $\{x(t): t \in[0, b]\}$ such that $x$ is continuous at $t \neq t_{i}, x\left(t_{i}\right)=x\left(t_{i}^{-}\right)$and $x\left(t_{i}^{+}\right)$exist for all $i=1, \ldots, N$. In this paper, we always assume that $\mathcal{P C}$ is endowed with the norm

$$
\|x\|_{\mathcal{P C}}=\left(\sup _{0 \leq t \leq b} E\|x(t)\|^{2}\right)^{\frac{1}{2}} .
$$

Then $\left(\mathcal{P C},\|\cdot\|_{\mathcal{P C}}\right)$ is a Banach space. The notation $B_{r}(x, H)$ stands for the closed ball with center at $x$ and radius $r>0$ in $H$.

Lemma 2.2 ([27, 28]) A family ofbounded linear operators $R(t) \in L(H)$ is called a resolvent operator for

$$
\frac{d x}{d t}=A\left[x(t)+\int_{0}^{t} h(t-s) x(s) d s\right]
$$

if

(i) $R(0)=I$ the identity operator on $H$;

(ii) for all $x \in H, R(t) x$ is continuous for $t \in[0, b]$;

(iii) $R(t) \in L(X), t \in J$, where $X$ is the Banach space formed from $D(R)$ endowed with the graph norm. For $y \in X, R(\cdot) y \in C^{1}(J, H) \cap C(J, X)$ and

$$
\begin{aligned}
\frac{d x}{d t} R(t) y & =A\left[R(t) y+\int_{0}^{t} h(t-s) R(s) y d s\right] \\
& =R(t) A y+\int_{0}^{t} R(t-s) A h(s) y d s, \quad t \in J .
\end{aligned}
$$

Let $0 \in \rho(A)$, Then it is possible to define the fractional power $(-A)^{\alpha}$ for $0<\alpha \leq 1$, as a closed linear operator on its domain $D\left((-A)^{\alpha}\right)$ with inverse $(-A)^{-\alpha}$. Furthermore, the subspace $D\left((-A)^{\alpha}\right)$ is dense in $H$ and the expression $\|x\|_{\alpha}=\left\|(-A)^{\alpha} x\right\|$ for $x \in D\left((-A)^{\alpha}\right)$. Defines a norm on $D\left((-A)^{\alpha}\right)$. Hereafter we denote by $H_{\alpha}$ the Banach space $D\left((-A)^{\alpha}\right)$ normed 
with $\|\cdot\|_{\alpha}$. Then, for each $0<\alpha \leq 1, H_{\alpha}$ is a Banach space. Furthermore, the following properties are well known.

Lemma 2.3 ([32]) The following properties hold:

(i) If $0<\beta<\alpha \leq 1$, then $H_{\alpha} \subset H_{\beta}$ and the embedding is compact whenever the resolvent operator of $A$ is compact.

(ii) For each $0<\alpha \leq 1$, there exists a positive constant $M_{\alpha}$ such that

$$
\left\|(-A)^{\alpha} R(t)\right\| \leq \frac{M_{\alpha}}{t^{\alpha}} .
$$

Definition 2.1 An $\mathcal{F}_{t}$-adapted stochastic process $x:(-\infty, b] \rightarrow H$ is called a mild solution of the system (1.1)-(1.3) if $x_{0}=\varphi \in \mathcal{B}$ satisfying $x_{0} \in L_{2}^{0}(\Omega, H),\left.x\right|_{[0, b]} \in \mathcal{P C}$, for each $t \in$ $[0, b]$, the function $A R(t-s) G\left(s, x_{s}\right), s \in[0, t)$ is integrable and $x(t)=g_{j}(t, x(t))$ for all $t \in$ $\left(t_{j}, s_{j}\right], j=1, \ldots, N$, and

$$
\begin{aligned}
x(t)= & R(t)[\varphi(0)-G(0, \varphi)]+G\left(t, x_{t}\right)+\int_{0}^{t} A R(t-s) G\left(s, x_{s}\right) d s \\
& +\int_{0}^{t} A R(t-s) \int_{0}^{s} h(s-\tau) G\left(\tau, x_{\tau}\right) d \tau d s+\int_{0}^{t} R(t-s) f\left(s, x_{s}\right) d s \\
& +\int_{0}^{t} R(t-s) F\left(s, x_{s}\right) d w(s)
\end{aligned}
$$

for all $t \in\left[0, t_{1}\right]$ and

$$
\begin{aligned}
x(t)= & R\left(t-s_{i}\right)\left[g_{i}\left(s_{i}, x\left(s_{i}\right)\right)-G\left(s_{i}, x_{s_{i}}\right)\right]+G\left(t, x_{t}\right)+\int_{s_{i}}^{t} A R(t-s) G\left(s, x_{s}\right) d s \\
& +\int_{s_{i}}^{t} A R(t-s) \int_{s_{i}}^{s} h(s-\tau) G\left(\tau, x_{\tau}\right) d \tau d s+\int_{s_{i}}^{t} R(t-s) f\left(s, x_{s}\right) d s \\
& +\int_{s_{i}}^{t} R(t-s) F\left(s, x_{s}\right) d w(s)
\end{aligned}
$$

for all $t \in\left(s_{i}, t_{i+1}\right], i=1, \ldots, N$.

Now, we introduce the Hausdorff measure of noncompactness $\chi_{Y}$ defined by

$$
\chi_{Y}(B)=\inf \{\varepsilon>0 ; B \text { has a finite } \varepsilon \text {-net in } H\}
$$

for a bounded set $B$ in any Hilbert space $Y$. Some basic properties of $\chi_{Y}(\cdot)$ are given in the following lemma.

Lemma 2.4 ([33]) Let $Y$ be a real Hilbert space and $B, C \subseteq Y$ be bounded, and the following properties are satisfied:

(1) $B$ is pre-compact if and only if $\chi_{Y}(B)=0$;

(2) $\chi_{Y}(B)=\chi_{Y}(\bar{B})=\chi_{Y}(\operatorname{conv} B)$, where $\bar{B}$ and $\operatorname{conv} B$ are the closure and the convex hull of $B$, respectively;

(3) $\chi_{Y}(B) \leq \chi_{Y}(C)$ when $B \subseteq C$;

(4) $\chi_{Y}(B+C) \leq \chi_{Y}(B)+\chi_{Y}(C)$ where $B+C=\{x+y: x \in B, y \in C\}$; 
(5) $\chi_{Y}(B \cup C)=\max \left\{\chi_{Y}(B), \chi_{Y}(C)\right\}$;

(6) $\chi_{Y}(\lambda B) \leq|\lambda| \chi_{Y}(B)$ for any $\lambda \in \mathbb{R}$;

(7) if the map $\Phi: D(\Phi) \subseteq Y \rightarrow Z$ is Lipschitz continuous with constant $\kappa$ then $\chi_{Z}(\Phi B) \leq \kappa \chi_{Y}(B)$ for any bounded subset $B \subseteq D(\Phi)$, where $Z$ is a Banach space;

(8) if $\left\{W_{n}\right\}_{n=1}^{\infty}$ is a decreasing sequence of bounded closed nonempty subset of $Y$ and $\lim _{n \rightarrow \infty} \chi_{Y}\left(W_{n}\right)=0$, then $\bigcap_{n=1}^{\infty} W_{n}$ is nonempty and compact in $Y$.

Definition 2.2 ([34]) The map $\Phi: W \subseteq Y \rightarrow Y$ is said to be a $\chi_{Y}$-contraction if there exists a positive constant $\kappa<1$ such that $\chi_{Y}(\Phi(C)) \leq \kappa \chi_{Y}(C)$ for any bounded close subset $C \subseteq W$ where $Y$ is a Banach space.

In this paper we denote by $\chi_{C}$ the Hausdorff measure of noncompactness of $C([0, b], H)$ and by $\chi_{\mathcal{P C}}$ the Hausdorff measure of noncompactness of $\mathcal{P C}$. To discuss the existence we need the following results.

Lemma 2.5 ([33]) If $W \subset C([a, b])$ is bounded, then $\chi(W(t)) \leq \chi_{C}(W)$, for any $t \in[a, b]$, where $W(t)=\{u(t): u \in W \subseteq X\}$. Furthermore, if $W$ is equicontinuous on $[a, b]$, then $W(t)$ is continuous for $t \in[a, b]$, and $\chi_{C}(W)=\sup \{W(t): t \in[a, b]\}$.

Lemma 2.6 ([33]) If $W \subset C([a, b], H)$ is bounded and equicontinuous, then $\chi(W(t))$ is continuous for $t \in[a, b]$, and $\chi\left(\int_{a}^{t} W(s) d s\right) \leq \int_{a}^{t} \chi(W(s)) d s$ for all $t \in[a, b]$, where $\int_{a}^{t} W(s) d s=\left\{\int_{a}^{t} x(s) d s: x \in W\right\}$.

Obviously, the following lemma holds.

Lemma 2.7 If $W \subset \mathcal{P C}([0, b], H)$ is bounded, then $\chi(W(t)) \leq \chi_{\mathcal{P C}}(W)$, for any $t \in[0, b]$, where $W(t)=\{u(t): u \in W \subseteq H\}$. Furthermore, if $W$ is piecewise equicontinuous on $[0, b]$, then $W(t)$ is continuous for $t \in[0, b]$, and $\chi_{\mathcal{P C}}(W)=\sup \{W(t): t \in[0, b]\}$.

Proof Let $W \subset \mathcal{P C}$, and $W=\left(\bigcup_{i=0}^{N} W_{i}\right) \cup\left(\bigcup_{i=1}^{N} U_{i}\right)$, where $W_{i} \subset C\left(\bar{J}_{i}, X\right), \bar{J}_{i}=\left[s_{i}, t_{i+1}\right], i=$ $0,1, \ldots, N$, and $U_{i} \subset C\left(\bar{I}_{i}, X\right), \bar{I}_{i}=\left[t_{i}, s_{i}\right], i=1, \ldots, N$. Using Lemmas 2.4 and 2.5 , we have

$$
\begin{aligned}
\chi(W(t)) & =\chi\left(\left(\bigcup_{i=0}^{N} W_{i}(t)\right) \cup\left(\bigcup_{i=1}^{N} U_{i}(t)\right)\right) \\
& =\max \left\{\max \left\{\chi\left(W_{i}(t)\right): i=0,1, \ldots, N\right\}, \max \left\{\chi\left(U_{i}(t)\right): i=1, \ldots, N\right\}\right\} \\
& \leq \max \left\{\max \left\{\chi_{\mathcal{C}}\left(W_{i}\right): i=0,1, \ldots, N\right\}, \max \left\{\chi_{\mathcal{C}}\left(U_{i}\right): i=1, \ldots, N\right\}\right\} \\
& =\max \left\{\max \left\{\chi_{\mathcal{P C}}\left(W_{i}\right): i=0,1, \ldots, N\right\}, \max \left\{\chi_{\mathcal{P C}}\left(U_{i}\right): i=1, \ldots, N\right\}\right\} \\
& =\max \left\{\chi_{\mathcal{P C}}\left(\bigcup_{i=0}^{N} W_{i}\right), \chi_{\mathcal{P C}}\left(\bigcup_{i=1}^{N} U_{i}\right)\right\} \\
& =\chi_{\mathcal{P C}}\left(\left(\bigcup_{i=0}^{N} W_{i}\right) \cup\left(\bigcup_{i=1}^{N} U_{i}\right)\right) \\
& =\chi_{\mathcal{P C}}(W) .
\end{aligned}
$$


From Lemma 2.5, if $W$ is piecewise equicontinuous on $[0, b]$, then $W(t)$ is continuous for $t \in[0, b]$. In addition, we have

$$
\begin{aligned}
\chi_{\mathcal{P C}}(W)= & \max \left\{\max \left\{\chi_{\mathcal{P C}}\left(W_{i}\right): i=0,1, \ldots, N\right\}, \max \left\{\chi_{\mathcal{P C}}\left(U_{i}\right): i=1, \ldots, N\right\}\right\} \\
= & \max \left\{\max \left\{\sup \left\{\chi\left(W_{i}(t)\right), t \in \bar{J}_{i}: i=0,1, \ldots, N\right\}\right\},\right. \\
& \left.\max \left\{\sup \left\{\chi\left(U_{i}(t)\right), t \in \bar{I}_{i}: i=1, \ldots, N\right\}\right\}\right\} \\
= & \max \{\sup \{\chi(W(t)), t \in[0, b]\}\}=\sup \{\chi(W(t)), t \in[0, b]\} .
\end{aligned}
$$

Lemma 2.8 (Darbo [35]) If $W \subseteq Y$ is closed and convex and $0 \in W$, the continuous map $\Phi: W \rightarrow W$ is a $\chi_{Y}$-contraction, if the set $\{x \in W: x=\lambda \Phi x\}$ is bounded for $0<\lambda<1$, then the map $\Phi$ has at least one fixed point in $W$.

Lemma 2.9 (Darbo-Sadovskii [33]) If $W \subseteq Y$ is bounded closed and convex, the continuous map $\Phi: W \rightarrow W$ is a $\chi_{Y}$-contraction, then the map $\Phi$ has at least one fixed point in $W$.

\section{Main results}

In this section we shall present and prove our main results. For some $\alpha \in(0,1)$, let us list the following hypotheses.

(H1) The analytic resolvent operator $R(t)$ generated by $A$, and there exist constants $M$, $M_{1}$ such that $\|R(t)\|_{L(H)} \leq M,\|h(t)\|_{L(H)} \leq M_{1}$ for every $t \geq 0$.

(H2) There exist positive constants $\beta \in\left(\frac{1}{2}, 1\right), L, L_{1}, L_{2}$ such that the function $G$ is $H_{\beta}$-valued, $(-A)^{\beta} G$ is continuous and

$$
\begin{aligned}
& E\left\|(-A)^{\beta} G\left(t, \psi_{1}\right)-(-A)^{\beta} G\left(t, \psi_{2}\right)\right\|^{2} \leq L\left\|\psi_{1}-\psi_{2}\right\|_{\mathcal{B}}^{2}, \quad t \in[0, b], \psi_{1}, \psi_{2} \in \mathcal{B}, \\
& E\left\|(-A)^{\beta} G(t, \psi)\right\|^{2} \leq L_{1}\|\psi\|_{\mathcal{B}}^{2}+L_{2}, \quad t \in[0, b], \psi \in \mathcal{B} .
\end{aligned}
$$

(H3) The function $f:[0, b] \times \mathcal{B} \rightarrow H$ satisfies the following conditions:

(i) For each $t \in[0, b]$, the function $f(t, \cdot): \mathcal{B} \rightarrow H$ is continuous and for each $x \in \mathcal{B}$, the function $f(\cdot, \psi):[0, b] \rightarrow H$ is strongly measurable.

(ii) There exist an integrable function $m_{f}:[0, b] \rightarrow[0, \infty)$ and a continuous nondecreasing function $\Theta_{f}:[0, \infty) \rightarrow(0, \infty)$ such that

$$
E\|f(t, \psi)\|^{2} \leq m_{f}(t) \Theta_{f}\left(\|\psi\|_{\mathcal{B}}^{2}\right), \quad t \in[0, b], \psi \in \mathcal{B}
$$

(iii) There exists an integrable function $\zeta_{f}:[0, b] \rightarrow[0, \infty)$ such that, for each bounded $D \subset \mathcal{B}$,

$$
\chi(f(t, D)) \leq \zeta_{f}(t) \sup _{-\infty<\theta \leq 0} \chi(D(\theta))
$$

for a.e. $t \in[0, b]$, where $D(\theta)=\{u(\theta): u \in D\}$.

(H4) The function $F:[0, b] \times \mathcal{B} \rightarrow L(K, H)$ satisfies the following conditions:

(i) For each $t \in[0, b]$, the function $F(t, \cdot): \mathcal{B} \rightarrow L(K, H)$ is continuous and for each $x \in \mathcal{B}$, the function $F(\cdot, \psi):[0, b] \rightarrow L(K, H)$ is strongly measurable. 
(ii) There exist an integrable function $m_{F}:[0, b] \rightarrow[0, \infty)$ and a continuous nondecreasing function $\Theta_{F}:[0, \infty) \rightarrow(0, \infty)$ such that

$$
E\|F(t, \psi)\|^{2} \leq m_{F}(t) \Theta_{F}\left(\|\psi\|_{\mathcal{B}}^{2}\right), \quad t \in[0, b], \psi \in \mathcal{B}
$$

(iii) There exists a square integrable function $\zeta_{F}:[0, b] \rightarrow[0, \infty)$ such that, for each bounded $D \subset \mathcal{B}$,

$$
\chi(F(t, D)) \leq \zeta_{F}(t) \sup _{-\infty<\theta \leq 0} \chi(D(\theta))
$$

for a.e. $t \in[0, b]$, where $D(\theta)=\{u(\theta): u \in D\}$.

(H5) The functions $g_{i}:\left(t_{i}, s_{i}\right] \times \mathcal{B} \rightarrow H, i=1, \ldots, N$, are continuous, and they satisfy the following conditions:

(i) There exist constants $c_{i}, d_{i}>0, i=1, \ldots, N$, such that

$$
E\left\|g_{i}(t, \psi)\right\|^{2} \leq c_{i}\|\psi\|_{\mathcal{B}}^{2}+d_{i}, \quad t \in\left(t_{i}, s_{i}\right], \psi \in \mathcal{B}
$$

(ii) There exist constants $\gamma_{i}>0$ such that, for each bounded $D \subset \mathcal{B}$,

$$
\chi\left(g_{i}(t, D)\right) \leq \gamma_{i} \sup _{-\infty<\theta \leq 0} \chi(D(\theta))
$$

for a.e. $t \in\left(t_{i}, s_{i}\right], i=1, \ldots, N$, where $D(\theta)=\{u(\theta): u \in D\}$, and

$$
\begin{aligned}
& \max _{1 \leq i \leq N}\left\{4 K_{b}\left(L\left[\left(1+M^{2}\right)\left\|(-A)^{-\beta}\right\|^{2}+M_{1-\beta}^{2}\left(1+M_{1}^{2} b^{2}\right) \frac{b^{2 \beta}}{2 \beta-1}\right]\right)^{\frac{1}{2}}\right. \\
& \left.\quad+(M+1) \gamma_{i}+M \int_{0}^{b} \zeta_{f}(s) d s+M\left(\operatorname{Tr}(Q) \int_{0}^{b} \zeta_{F}^{2}(s) d s\right)^{\frac{1}{2}}\right\}<1 .
\end{aligned}
$$

(H6) For every bounded set $\Xi \subset \mathcal{B}$, the set $\left\{t \rightarrow g_{i}\left(t, x_{t}\right): x_{t} \in \Xi\right\}, i=1, \ldots, N$, is equicontinuous in $\mathcal{B}$.

Theorem 3.1 If the assumptions (H1)-(H6) are satisfied, then the system (1.1)-(1.3) has at least one mild solution on $[0, b]$, provided that

$$
\int_{1}^{\infty} \frac{1}{\Theta_{f}(s)+\Theta_{F}(s)} d s=\infty
$$

and

$$
\begin{aligned}
& \max _{1 \leq i \leq N}\left\{4 K _ { b } ^ { 2 } \left(\left(1+12 M^{2}\right) c_{i}+\left[\left(1+12 M^{2}\right)\left\|(-A)^{-\beta}\right\|^{2}\right.\right.\right. \\
& \left.\left.\left.+6 M_{1-\beta}^{2}\left(1+M_{1}^{2} b^{2}\right) \frac{b^{2 \beta}}{2 \beta-1}\right] L_{1}\right)\right\}<1 .
\end{aligned}
$$

Proof We introduce the space $\mathcal{B}_{b}$ of all functions $x:(-\infty, b] \rightarrow H$ such that $x_{0} \in \mathcal{B}$ and the restriction $\left.x\right|_{[0, b]} \in \mathcal{P C}$. Let $\|\cdot\|_{b}$ be a seminorm in $\mathcal{B}_{b}$ defined by

$$
\|x\|_{b}=\left\|x_{0}\right\|_{\mathcal{B}}+\left(\sup _{0 \leq t \leq b} E\|x(t)\|^{2}\right)^{\frac{1}{2}}, \quad x \in \mathcal{B}_{b}
$$


We consider the operator $\Phi: \mathcal{B}_{b} \rightarrow \mathcal{B}_{b}$ defined by

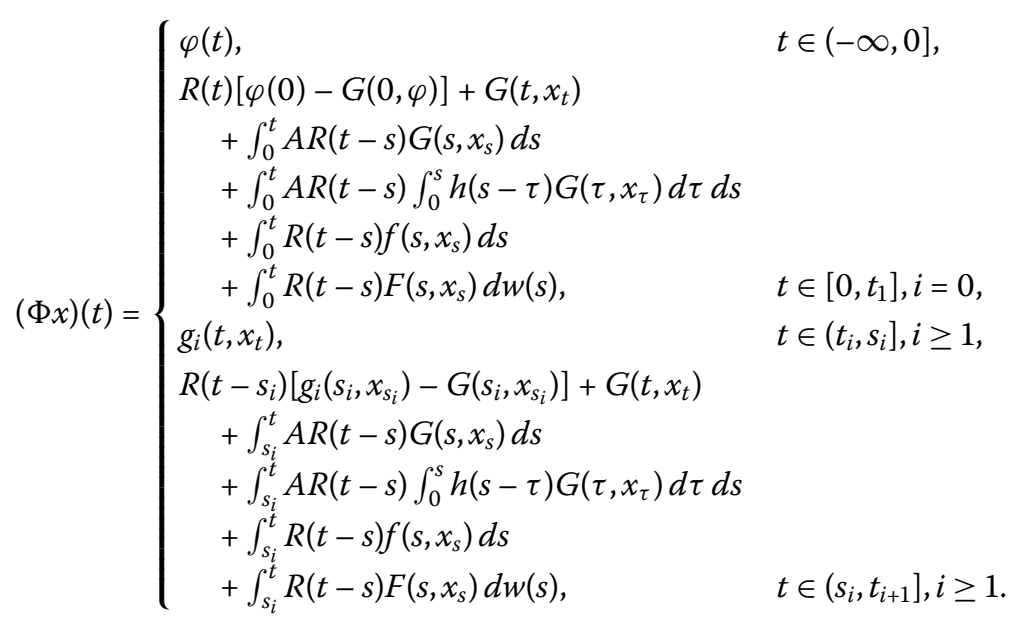

From Lemma 2.3 and (H2), the following inequality holds:

$$
\begin{aligned}
E & \left\|\int_{s_{i}}^{t} A R(t-s) G\left(s, x_{s}\right) d s\right\|^{2} \\
& \leq b \int_{s_{i}}^{t}\left\|(-A)^{1-\beta} R(t-s)\right\|^{2} E\left\|(-A)^{\beta} G\left(s, x_{s}\right)\right\|^{2} d s \\
& \leq b M_{1-\beta}^{2} \int_{s_{i}}^{t}(t-s)^{-2(1-\beta)}\left(L_{1}\left\|x_{s}\right\|_{\mathcal{B}}^{2}+L_{2}\right) d s \\
& \leq M_{1-\beta}^{2} \frac{b^{2 \beta}}{2 \beta-1}\left(L_{1} \sup _{s \in[0, b]}\left\|x_{s}\right\|_{\mathcal{B}}^{2}+L_{2}\right) .
\end{aligned}
$$

Then from the Bochner theorem, it follows that $A \mathcal{S}_{\alpha}(t-s) G\left(s, x_{s}\right)$ is integrable on $\left(s_{i}, t\right)$, $i=0,1, \ldots, N$.

For $\varphi \in \mathcal{B}$, we define $\tilde{\varphi}$ by

$$
\tilde{\varphi}(t)= \begin{cases}\varphi(t), & -\infty<t \leq 0 \\ R(t) \varphi(0), & 0 \leq t \leq b\end{cases}
$$

then $\tilde{\varphi} \in \mathcal{B}_{b}$. Set $x(t)=y(t)+\tilde{\varphi}(t),-\infty<t \leq b$. It is clear that $x$ satisfies Definition 2.1 if and only if $y$ satisfies $y_{0}=0$ and

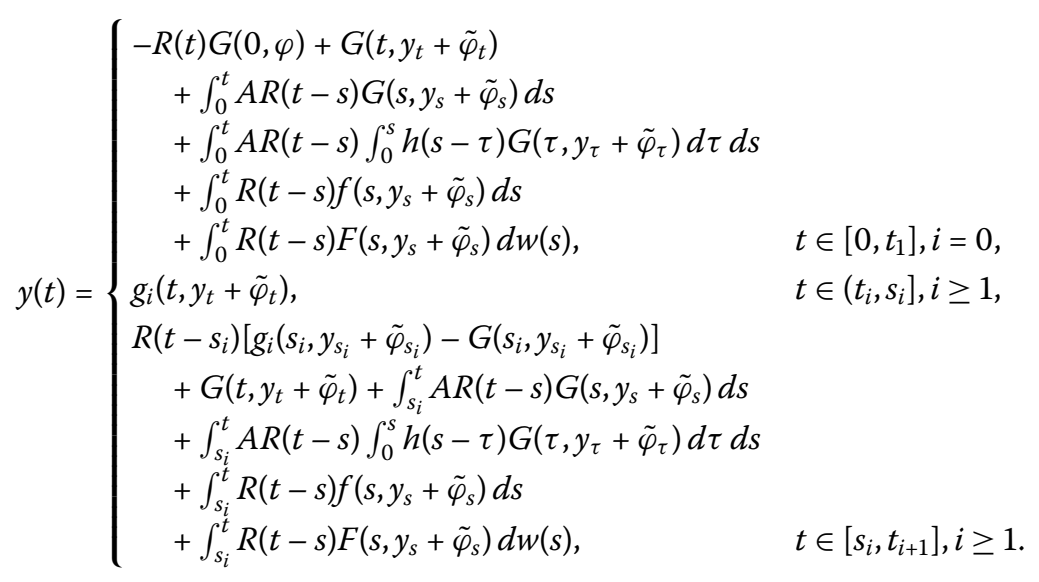


Let $\mathcal{B}_{b}^{0}=\left\{y \in \mathcal{B}_{b}: y_{0}=0 \in \mathcal{B}\right\}$. For any $y \in \mathcal{B}_{b}^{0}$,

$$
\|y\|_{b}=\left\|y_{0}\right\|_{\mathcal{B}}+\left(\sup _{0 \leq t \leq b} E\|y(t)\|^{2}\right)^{\frac{1}{2}}=\left(\sup _{0 \leq t \leq b} E\|y(t)\|^{2}\right)^{\frac{1}{2}} .
$$

By (3.4), it follows that $\left(\mathcal{B}_{b}^{0},\|\cdot\|_{b}\right)$ is a Banach space. For $y \in B_{r}\left(0, \mathcal{B}_{b}^{0}\right)=\left\{y \in \mathcal{B}_{b}^{0}: E\|y\|^{2} \leq r\right\}$, from Lemma 2.1, we have

$$
\begin{aligned}
\left\|y_{t}+\tilde{\varphi}_{t}\right\|_{\mathcal{B}}^{2} & \leq 2\left(\left\|y_{t}\right\|_{\mathcal{B}}^{2}+\left\|\tilde{\varphi}_{t}\right\|_{\mathcal{B}}^{2}\right) \\
& \leq 4\left[K_{b}^{2} E\left(\sup _{0 \leq s \leq b}\|y(s)\|^{2}\right)+M_{b}^{2} E\left\|y_{0}\right\|_{\mathcal{B}}^{2}+K_{b}^{2} E\left(\sup _{0 \leq s \leq b}\|\tilde{\varphi}(s)\|^{2}\right)+M_{b}^{2}\left\|\tilde{\varphi}_{0}\right\|_{\mathcal{B}}^{2}\right] \\
& \leq 4\left[K_{b}^{2} \sup _{0 \leq s \leq b} E\|y(s)\|^{2}+K_{b}^{2} M^{2} E\|\varphi(0)\|^{2}+M_{b}^{2}\|\varphi\|_{\mathcal{B}}^{2}\right] \\
& \leq 4\left[K_{b}^{2} r+\left(K_{b}^{2} M^{2} \tilde{H}^{2}+M_{b}^{2}\right)\|\varphi\|_{\mathcal{B}}^{2}\right]=r^{*}, \quad t \in[0, b]
\end{aligned}
$$

Define the map $\bar{\Phi}: \mathcal{B}_{b}^{0} \rightarrow \mathcal{B}_{b}^{0}$ defined by $(\bar{\Phi} y)(t)=0, t \in(-\infty, 0]$ and

$$
(\bar{\Phi} y)(t)=\left\{\begin{array}{rrr}
-R & (t) G(0, \varphi)+G\left(t, y_{t}+\tilde{\varphi}_{t}\right) & \\
& +\int_{0}^{t} A R(t-s) G\left(s, y_{s}+\tilde{\varphi}_{s}\right) d s & \\
& +\int_{0}^{t} A R(t-s) \int_{0}^{s} h(s-\tau) G\left(\tau, y_{\tau}+\tilde{\varphi}_{\tau}\right) d \tau d s & \\
& +\int_{0}^{t} R(t-s) f\left(s, y_{s}+\tilde{\varphi}_{s}\right) d s & \\
& +\int_{0}^{t} R(t-s) F\left(s, y_{s}+\tilde{\varphi}_{s}\right) d w(s), & \\
g_{i}\left(t, y_{t}+\tilde{\varphi}_{t}\right), & & \\
R\left(t \in\left[t_{i}, s_{i}\right], i \geq 1,\right. & \\
& \left.+s_{i}\right)\left[g_{i}\left(s_{i}, y_{s_{i}}+\tilde{\varphi}_{s_{i}}\right)-G\left(s_{i}, y_{s_{i}}+\tilde{\varphi}_{s_{i}}\right)\right] & \\
& +G\left(t, y_{t}+\tilde{\varphi}_{t}\right)+\int_{s_{i}}^{t} A R(t-s) G\left(s, y_{s}+\tilde{\varphi}_{s}\right) d s & \\
& +\int_{s_{i}}^{t} A R(t-s) \int_{0}^{s} h(s-\tau) G\left(\tau, y_{\tau}+\tilde{\varphi}_{\tau}\right) d \tau d s & \\
& +\int_{s_{i}}^{t} R(t-s) f\left(s, y_{s}+\tilde{\varphi}_{s}\right) d s & \\
& +\int_{s_{i}}^{t} R(t-s) F\left(s, y_{s}+\tilde{\varphi}_{s}\right) d w(s), & t \in\left[s_{i}, t_{i+1}\right], i \geq 1 .
\end{array}\right.
$$

Obviously, the operator $\Phi$ has a fixed point if and only if operator $\bar{\Phi}$ has a fixed point, to prove it we shall employ Lemma 2.8. For better readability, we break the proof into a sequence of steps.

Step 1 . For $0<\lambda<1$, set $\{y \in \mathcal{P C}: y=\lambda \bar{\Phi} y\}$ is bounded.

Let $y_{\lambda}$ be a solution of $y=\lambda \bar{\Phi} y$ for $0<\lambda<1$. Using Lemma 2.1, we obtain, for all $t \in[0, b]$,

$$
\sup _{0 \leq s \leq t}\left\|y_{\lambda t}+\tilde{\varphi}_{t}\right\|_{\mathcal{B}}^{2} \leq 4\left[K_{b}^{2} E\left\|y_{\lambda}\right\|_{t}^{2}+\left(K_{b}^{2} M^{2} \tilde{H}^{2}+M_{b}^{2}\right)\|\varphi\|_{\mathcal{B}}^{2}\right]
$$

where $\left\|y_{\lambda}\right\|_{t}=\sup _{0 \leq s \leq t}\left\|y_{\lambda}(s)\right\|$. Consider the function $v_{\lambda}$ defined by

$$
\nu_{\lambda}(t)=4\left[K_{b}^{2} E\left\|y_{\lambda}\right\|_{t}^{2}+\left(K_{b}^{2} M^{2} \tilde{H}^{2}+M_{b}^{2}\right)\|\varphi\|_{\mathcal{B}}^{2}\right], \quad 0 \leq t \leq b .
$$

Then, by (H1)-(H5), from the above equation, we have, for $t \in\left[0, t_{1}\right]$,

$$
\begin{aligned}
E\left\|y_{\lambda}(t)\right\|^{2} \leq & 6 E\|R(t) G(0, \varphi)\|^{2}+6 E\left\|G\left(t, y_{\lambda t}+\tilde{\varphi}_{t}\right)\right\|^{2} \\
& +6 E\left\|\int_{0}^{t} A R(t-s) G\left(s, y_{\lambda s}+\tilde{\varphi}_{s}\right) d s\right\|^{2}
\end{aligned}
$$




$$
\begin{aligned}
& +6 E\left\|\int_{0}^{t} A R(t-s) \int_{0}^{s} h(s-\tau) G\left(\tau, y_{\lambda \tau}+\tilde{\varphi}_{\tau}\right) d \tau d s\right\|^{2} \\
& +6 E\left\|\int_{0}^{t} R(t-s) f\left(s, y_{\lambda s}+\tilde{\varphi}_{s}\right) d s\right\|^{2} \\
& +6 E\left\|\int_{0}^{t} R(t-s) F\left(s, y_{\lambda s}+\tilde{\varphi}_{s}\right) d w(s)\right\|^{2} \\
& \leq 6 M^{2}\left\|(-A)^{-\beta}\right\|^{2}\left(L_{1}\|\varphi\|_{\mathcal{B}}^{2}+L_{2}\right)+\left\|(-A)^{-\beta}\right\|^{2}\left(L_{1} v_{\lambda}(t)+L_{2}\right) \\
& +6 M_{1-\beta}^{2} t_{1} \int_{0}^{t}(t-s)^{-2(1-\beta)}\left(L_{1} v_{\lambda}(s)+L_{2}\right) d s \\
& +6 M_{1-\beta}^{2} M_{1}^{2} t_{1}^{2} \int_{0}^{t}(t-s)^{-2(1-\beta)} \int_{0}^{s}\left(L_{1} v_{\lambda}(\tau)+L_{2}\right) d \tau d s \\
& +6 M^{2} t_{1} \int_{0}^{t} m_{f}(s) \Theta_{f}\left(v_{\lambda}(s)\right) d s \\
& +6 M^{2} \operatorname{Tr}(Q) \int_{0}^{t} m_{F}(s) \Theta_{F}\left(v_{\lambda}(s)\right) d s .
\end{aligned}
$$

For any $t \in\left(t_{i}, s_{i}\right], i=1, \ldots, N$, we have

$$
E\left\|y_{\lambda}(t)\right\|^{2}=E\left\|g_{i}\left(t, y_{t}+\tilde{\varphi}_{t}\right)\right\|^{2} \leq c_{i} v_{\lambda}(t)+d_{i}
$$

Similarly, for any $t \in\left(s_{i}, t_{i+1}\right], i=1, \ldots, N$, we have

$$
\begin{aligned}
E\left\|y_{\lambda}(t)\right\|^{2} \leq & 6 E\left\|R\left(t-s_{i}\right)\left[g_{i}\left(s_{i}, y_{\lambda s_{i}}+\tilde{\varphi}_{s_{i}}\right)-G\left(s_{i}, y_{\lambda s_{i}}+\tilde{\varphi}_{s_{i}}\right)\right]\right\|^{2} \\
& +6 E\left\|G\left(t, y_{\lambda t}+\tilde{\varphi}_{t}\right)\right\|^{2}+6 E\left\|\int_{s_{i}}^{t} A R(t-s) G\left(s, y_{\lambda s}+\tilde{\varphi}_{s}\right) d s\right\|^{2} \\
& +6 E\left\|\int_{s_{i}}^{t} A R(t-s) \int_{0}^{s} h(s-\tau) G\left(\tau, y_{\lambda \tau}+\tilde{\varphi}_{\tau}\right) d \tau d s\right\|^{2} \\
& +6 E\left\|\int_{s_{i}}^{t} R(t-s) f\left(s, y_{\lambda s}+\tilde{\varphi}_{s}\right) d s\right\|^{2} \\
& +6 E\left\|\int_{s_{i}}^{t} R(t-s) F\left(s, y_{\lambda s}+\tilde{\varphi}_{s}\right) d w(s)\right\|^{2} \\
\leq & 12 M^{2}\left[c_{i} v_{\lambda}\left(s_{i}\right)+d_{i}+\left\|(-A)^{-\beta}\right\|^{2}\left(L_{1} v_{\lambda}\left(s_{i}\right)+L_{2}\right)\right] \\
& +6\left\|(-A)^{-\beta}\right\|^{2}\left(L_{1} v_{\lambda}(t)+L_{2}\right) \\
& +6 M_{1-\beta}^{2}\left(t_{i+1}-s_{i}\right) \int_{s_{i}}^{t}(t-s)^{-2(1-\beta)}\left(L_{1} v_{\lambda}(s)+L_{2}\right) d s \\
& +6 M_{1-\beta}^{2} M_{1}^{2}\left(t_{i+1}-s_{i}\right)^{2} \int_{0}^{t}(t-s)^{-2(1-\beta)} \int_{s_{i}}^{s}\left(L_{1} v_{\lambda}(\tau)+L_{2}\right) d \tau d s \\
& +6 M^{2}\left(t_{i+1}-s_{i}\right) \int_{s_{i}}^{t} m_{f}(s) \Theta_{f}\left(v_{\lambda}(s)\right) d s \\
& +6 M^{2} \operatorname{Tr}(Q) \int_{s_{i}}^{t} m_{F}(s) \Theta_{F}\left(v_{\lambda}(s)\right) d s .
\end{aligned}
$$


Then, for all $t \in[0, b]$, we have

$$
\begin{aligned}
E\left\|y_{\lambda}(t)\right\|^{2} \leq & M^{*}+c_{i} v_{\lambda}(t)+12 M^{2}\left[c_{i} v_{\lambda}(t)+\left\|(-A)^{-\beta}\right\|^{2} L_{1} v_{\lambda}(t)\right] \\
& +6\left\|(-A)^{-\beta}\right\|^{2} L_{1} v_{\lambda}(t) \\
& +6 M_{1-\beta}^{2} b \int_{0}^{t}(t-s)^{-2(1-\beta)} L_{1} v_{\lambda}(s) d s \\
& +6 M_{1-\beta}^{2} M_{1}^{2} b^{2} \int_{0}^{t}(t-s)^{-2(1-\beta)} \int_{0}^{s} L_{1} v_{\lambda}(\tau) d \tau d s \\
& +6 M^{2} b \int_{0}^{t} m_{f}(s) \Theta_{f}\left(v_{\lambda}(s)\right) d s \\
& +6 M^{2} \operatorname{Tr}(Q) \int_{0}^{t} m_{F}(s) \Theta_{F}\left(v_{\lambda}(s)\right) d s,
\end{aligned}
$$

where

$$
\begin{aligned}
M^{*}= & \max _{1 \leq i<N}\left\{6 M^{2}\left\|(-A)^{-\beta}\right\|^{2}\left(L_{1}\|\varphi\|_{\mathcal{B}}^{2}+L_{2}\right)+\left(1+12 M^{2}\right) d_{i}\right. \\
& \left.+\left[\left(1+12 M^{2}\right)\left\|(-A)^{-\beta}\right\|^{2}+6 M_{1-\beta}^{2}\left(1+M_{1}^{2} b^{2}\right) \frac{b^{2 \beta}}{2 \beta-1}\right] L_{2}\right\} .
\end{aligned}
$$

It is easy to see that

$$
\begin{aligned}
v_{\lambda}(t) \leq & 4\left[K_{b}^{2} M^{*}+\left(K_{b}^{2} M^{2} \tilde{H}^{2}+M_{b}^{2}\right)\|\varphi\|_{\mathcal{B}}^{2}\right]+4 K_{b}^{2}\left(\left(1+12 M^{2}\right) c_{i}\right. \\
& \left.+\left[\left(1+12 M^{2}\right)\left\|(-A)^{-\beta}\right\|^{2}+6 M_{1-\beta}^{2}\left(1+M_{1}^{2} b^{2}\right) \frac{b^{2 \beta}}{2 \beta-1}\right] L_{1}\right) v_{\lambda}(t) \\
& +24 K_{b}^{2} M^{2} b \int_{0}^{t} m_{f}(s) \Theta_{f}\left(v_{\lambda}(s)\right) d s \\
& +24 K_{b}^{2} M^{2} \operatorname{Tr}(Q) \int_{0}^{t} m_{F}(s) \Theta_{F}\left(v_{\lambda}(s)\right) d s .
\end{aligned}
$$

By (3.3), we have

$$
\begin{aligned}
L^{*}= & \max _{1 \leq i \leq N}\left\{4 K _ { b } ^ { 2 } \left(\left(1+12 M^{2}\right) c_{i}+\left[\left(1+12 M^{2}\right)\left\|(-A)^{-\beta}\right\|^{2}\right.\right.\right. \\
& \left.\left.\left.+6 M_{1-\beta}^{2}\left(1+M_{1}^{2} b^{2}\right) \frac{b^{2 \beta}}{2 \beta-1}\right] L_{1}\right)\right\}<1,
\end{aligned}
$$

and hence

$$
\begin{aligned}
v_{\lambda}(t) \leq & \frac{4\left[K_{b}^{2} M^{*}+\left(K_{b}^{2} M^{2} \tilde{H}^{2}+M_{b}^{2}\right)\|\varphi\|_{\mathcal{B}}^{2}\right]}{1-L^{*}} \\
& +\frac{24 K_{b}^{2} M^{2} b}{1-L^{*}} \int_{0}^{t} m_{f}(s) \Theta_{f}\left(v_{\lambda}(s)\right) d s \\
& +\frac{24 K_{b}^{2} M^{2} \operatorname{Tr}(Q)}{1-L^{*}} \int_{0}^{t} m_{F}(s) \Theta_{F}\left(v_{\lambda}(s)\right) d s .
\end{aligned}
$$


Denoting by $\eta_{\lambda}(t)$ the right-hand side of the above inequality, we have $v_{\lambda}(t) \leq \eta_{\lambda}(t)$ for all $t \in[0, b]$, and

$$
\begin{aligned}
\eta_{\lambda}(0) & =\frac{4\left[K_{b}^{2} M^{*}+\left(K_{b}^{2} M^{2} \tilde{H}^{2}+M_{b}^{2}\right)\|\varphi\|_{\mathcal{B}}^{2}\right]}{1-L^{*}}, \\
\eta_{\lambda}^{\prime}(t) & \leq \frac{24 K_{b}^{2} M^{2} b}{1-L^{*}} m_{f}(t) \Theta_{f}\left(\eta_{\lambda}(t)\right)+\frac{24 K_{b}^{2} M^{2} \operatorname{Tr}(Q)}{1-L^{*}} m_{F}(t) \Theta_{F}\left(\eta_{\lambda}(t)\right) \\
& \leq \max \left\{\frac{24 K_{b}^{2} M^{2} b}{1-L^{*}} m_{f}(t), \frac{24 K_{b}^{2} M^{2} \operatorname{Tr}(Q)}{1-L^{*}} m_{F}(t)\right\}\left[\Theta_{f}\left(\eta_{\lambda}(t)\right)+\Theta_{F}\left(\eta_{\lambda}(t)\right)\right] .
\end{aligned}
$$

This implies that

$$
\int_{\eta_{\lambda}(0)}^{\eta_{\lambda}(t)} \frac{d s}{\Theta_{f}(s)+\Theta_{F}(s)} \leq \int_{0}^{b} \max \left\{\frac{24 K_{b}^{2} M^{2} b}{1-L^{*}} m_{f}(t), \frac{24 K_{b}^{2} M^{2} \operatorname{Tr}(Q)}{1-L^{*}} m_{F}(t)\right\} d t<\infty .
$$

Using (3.2), this inequality shows that the functions $\eta_{\lambda}(t)$ are bounded on $[0, b]$. Thus, the functions $v_{\lambda}(t)$ are bounded on $[0, b]$, and $y_{\lambda}(\cdot)$ are bounded on $[0, b]$.

Step 2. $\bar{\Phi}: \mathcal{B}_{b}^{0} \rightarrow \mathcal{B}_{b}^{0}$ is continuous.

Let $\left\{y^{(n)}\right\}_{n=0}^{\infty} \subseteq \mathcal{B}_{b}^{0}$ with $y^{(n)} \rightarrow y(n \rightarrow \infty)$ in $\mathcal{B}_{b}^{0}$. Then there is a number $r>0$ such that $\left\|y^{(n)}(t)\right\| \leq r$ for all $n$ and a.e. $t \in[0, b]$, so $y^{(n)} \in B_{r}\left(0, \mathcal{B}_{b}^{0}\right)$ and $y \in B_{r}\left(0, \mathcal{B}_{b}^{0}\right)$. Again, similarly to (3.5), we have $\left\|y_{t}^{(n)}+\tilde{\varphi}_{t}\right\|_{\mathcal{B}} \leq r^{*}, t \in[0, b]$. Furthermore, from axiom (A), we know that

$$
\begin{aligned}
\left\|y_{t}^{(n)}-y_{t}\right\|_{\mathcal{B}} & \leq K(t) \sup \left\{\left\|y^{n}(s)-y(s)\right\|: 0 \leq s \leq t\right\}+M(t)\left\|y_{0}^{(n)}-y_{0}\right\|_{\mathcal{B}} \\
& =K(t) \sup \left\{\left\|y^{n}(s)-y(s)\right\|: 0 \leq s \leq t\right\} \\
& \leq K_{b}\left\|y^{n}-y\right\|_{b} \rightarrow 0 \quad(n \rightarrow \infty), t \in[0, b] .
\end{aligned}
$$

Thus, by (H3) and (H4), we see that, for $t \in[0, b]$,

$$
\begin{array}{r}
f\left(t, y_{t}^{(n)}+\tilde{\varphi}_{t}\right) \rightarrow f\left(t, y_{t}+\tilde{\varphi}_{t}\right) \quad \text { as } n \rightarrow \infty, \\
F\left(t, y_{t}^{(n)}+\tilde{\varphi}_{t}\right) \rightarrow F\left(t, y_{t}+\tilde{\varphi}_{t}\right) \quad \text { as } n \rightarrow \infty
\end{array}
$$

for each $t \in[0, b]$, and since

$$
\begin{aligned}
& E\left\|f\left(t, y_{t}^{(n)}+\tilde{\varphi}_{t}\right)-f\left(t, y_{t}+\tilde{\varphi}_{t}\right)\right\|^{2} \leq 2 \Theta_{f}\left(r^{*}\right) m_{f}(t), \\
& E\left\|F\left(t, y_{t}^{(n)}+\tilde{\varphi}_{t}\right)-F\left(t, y_{t}+\tilde{\varphi}_{t}\right)\right\|^{2} \leq 2 \Theta_{F}\left(r^{*}\right) m_{F}(t) .
\end{aligned}
$$

In view of (H2)-(H6) and by the dominated convergence theorem we have, for $t \in\left[0, t_{1}\right]$,

$$
\begin{aligned}
E \|( & \left(\bar{\Phi} y^{(n)}\right)(t)-(\bar{\Phi} y)(t) \|^{2} \\
\leq & 5\left\|(-A)^{-\beta}\right\| E\left\|(-A)^{\beta} G\left(t, y_{t}^{(n)}+\tilde{\varphi}_{t}\right)-(-A)^{\beta} G\left(t, y_{t}+\tilde{\varphi}_{t}\right)\right\|^{2} \\
& +5 M_{1-\beta}^{2} t_{1} \int_{0}^{t}(t-s)^{-2(1-\beta)} E\left\|(-A)^{\beta} G\left(s, y_{s}^{(n)}+\tilde{\varphi}_{s}\right)-(-A)^{\beta} G\left(s, y_{s}+\tilde{\varphi}_{s}\right)\right\|^{2} d s \\
& +5 M_{1-\beta}^{2} M_{1}^{2} t_{1}^{2} \int_{0}^{t}(t-s)^{-2(1-\beta)} \int_{0}^{s} E \|(-A)^{\beta} G\left(\tau, y_{\tau}^{(n)}+\tilde{\varphi}_{\tau}\right)
\end{aligned}
$$




$$
\begin{aligned}
& -(-A)^{\beta} G\left(\tau, y_{\tau}+\tilde{\varphi}_{\tau}\right) \|^{2} d \tau d s \\
& +5 M^{2} t_{1} \int_{0}^{t} E\left\|f\left(s, y_{s}^{(n)}+\tilde{\varphi}_{s}\right)-f\left(s, y_{s}+\tilde{\varphi}_{s}\right)\right\|^{2} d s \\
& +5 M^{2} \operatorname{Tr}(Q) \int_{0}^{t} E\left\|F\left(s, y_{s}^{(n)}+\tilde{\varphi}_{s}\right)-F\left(s, y_{s}+\tilde{\varphi}_{s}\right)\right\|^{2} d s \rightarrow 0 \quad \text { as } n \rightarrow \infty
\end{aligned}
$$

For any $t \in\left(t_{i}, s_{i}\right], i=1, \ldots, N$, we have

$$
E\left\|\left(\bar{\Phi} y^{(n)}\right)(t)-(\bar{\Phi} y)(t)\right\|^{2}=E\left\|g_{i}\left(t, y_{t}^{(n)}+\tilde{\varphi}_{t}\right)-g_{i}\left(t, y_{t}+\tilde{\varphi}_{t}\right)\right\|^{2} \rightarrow 0 \quad \text { as } n \rightarrow \infty .
$$

Similarly, for any $t \in\left(s_{i}, t_{i+1}\right], i=1, \ldots, N$, we have

$$
\begin{aligned}
E \|( & \left.\bar{\Phi}^{(n)}\right)(t)-(\bar{\Phi} y)(t) \|^{2} \\
\leq & 12 M\left[E\left\|g_{i}\left(s_{i}, y_{s_{i}}^{(n)}+\tilde{\varphi}_{s_{i}}\right)-g_{i}\left(s_{i}, y_{s_{i}}+\tilde{\varphi}_{s_{i}}\right)\right\|^{2}\right. \\
& \left.+\left\|(-A)^{-\beta}\right\|^{2} E\left\|(-A)^{\beta} G\left(s_{i}, y_{s_{i}}^{(n)}+\tilde{\varphi}_{s_{i}}\right)-(-A)^{\beta} G\left(s_{i}, y_{s_{i}}+\tilde{\varphi}_{s_{i}}\right)\right\|^{2}\right] \\
& +6\left\|(-A)^{-\beta}\right\|^{2} E\left\|(-A)^{\beta} G\left(t, y_{t}^{(n)}+\tilde{\varphi}_{t}\right)-(-A)^{\beta} G\left(t, y_{t}+\tilde{\varphi}_{t}\right)\right\|^{2} \\
& +6 M_{1-\beta}^{2}\left(t_{i+1}-s_{i}\right) \int_{s_{i}}^{t}(t-s)^{-2(1-\beta)} E \|(-A)^{\beta} G\left(s, y_{s}^{(n)}+\tilde{\varphi}_{s}\right) \\
& -(-A)^{\beta} G\left(s, y_{s}+\tilde{\varphi}_{s}\right) \|^{2} d s \\
& +6 M_{1-\beta}^{2} M_{1}^{2}\left(t_{i+1}-s_{i}\right)^{2} \int_{s_{i}}^{t}(t-s)^{-2(1-\beta)} \int_{0}^{s} E \|(-A)^{\beta} G\left(\tau, y_{\tau}^{(n)}+\tilde{\varphi}_{\tau}\right) \\
& -(-A)^{\beta} G\left(\tau, y_{\tau}+\tilde{\varphi}_{\tau}\right) \|^{2} d \tau d s \\
& +6 M^{2}\left(t_{i+1}-s_{i}\right) \int_{s_{i}}^{t} E\left\|f\left(s, y_{s}^{(n)}+\tilde{\varphi}_{s}\right)-f\left(s, y_{s}+\tilde{\varphi}_{s}\right)\right\|^{2} d s \\
& +6 M^{2} \operatorname{Tr}(Q) \int_{s_{i}}^{t} E\left\|F\left(s, y_{s}^{(n)}+\tilde{\varphi}_{s}\right)-F\left(s, y_{s}+\tilde{\varphi}_{s}\right)\right\|^{2} d s \rightarrow 0 \quad \text { as } n \rightarrow \infty .
\end{aligned}
$$

Then

$$
\left\|\bar{\Phi} y^{(n)}-\bar{\Phi} y\right\|_{b}^{2} \rightarrow 0 \quad \text { as } n \rightarrow \infty \text {. }
$$

Thus $\bar{\Phi}$ is continuous.

Step 3. $\bar{\Phi}$ maps bounded sets into bounded sets in $\mathcal{B}_{b}^{0}$.

Indeed, it is enough to show that there exists a positive constant $\mathcal{L}$ such that each $y \in$ $B_{r}\left(0, \mathcal{B}_{b}^{0}\right)$, one has $E\|\bar{\Phi} y\|^{2} \leq \mathcal{L}$. For $t \in\left[0, t_{1}\right]$, we have

$$
\begin{aligned}
E\|(\bar{\Phi} y)(t)\|^{2} \leq & 6 M^{2}\left\|(-A)^{-\beta}\right\|^{2}\left(L_{1}\|\varphi\|_{\mathcal{B}}^{2}+L_{2}\right)+\left\|(-A)^{-\beta}\right\|^{2}\left(L_{1}\left\|y_{t}+\tilde{\varphi}_{t}\right\|_{\mathcal{B}}^{2}+L_{2}\right) \\
& +6 M_{1-\beta}^{2} t_{1} \int_{0}^{t}(t-s)^{-2(1-\beta)}\left(L_{1}\left\|y_{s}+\tilde{\varphi}_{s}\right\|_{\mathcal{B}}^{2}+L_{2}\right) d s \\
& +6 M_{1-\beta}^{2} M_{1}^{2} t_{1}^{2} \int_{0}^{t}(t-s)^{-2(1-\beta)} \int_{0}^{s}\left(L_{1}\left\|y_{\tau}+\tilde{\varphi}_{\tau}\right\|_{\mathcal{B}}^{2}+L_{2}\right) d \tau d s \\
& +6 M^{2} t_{1} \int_{0}^{t} m_{f}(s) \Theta_{f}\left(\left\|y_{s}+\tilde{\varphi}_{s}\right\|_{\mathcal{B}}^{2}\right) d s
\end{aligned}
$$




$$
\begin{aligned}
& +6 M^{2} \operatorname{Tr}(Q) \int_{0}^{t} m_{F}(s) \Theta_{F}\left(\left\|y_{s}+\tilde{\varphi}_{s}\right\|_{\mathcal{B}}^{2}\right) d s \\
\leq & 6 M^{2}\left\|(-A)^{-\beta}\right\|^{2}\left(L_{1}\|\varphi\|_{\mathcal{B}}^{2}+L_{2}\right)+\left\|(-A)^{-\beta}\right\|^{2}\left(L_{1} r^{*}+L_{2}\right) \\
& +6 M_{1-\beta}^{2}\left(1+M_{1}^{2} t_{1}^{2}\right) \frac{t_{1}^{2 \beta}}{2 \beta-1}\left(L_{1} r^{*}+L_{2}\right) \\
& +6 M^{2} t_{1} \Theta_{f}\left(r^{*}\right) \int_{0}^{t_{1}} m_{f}(s) d s+6 M^{2} \operatorname{Tr}(Q) \Theta_{F}\left(r^{*}\right) \int_{0}^{t_{1}} m_{F}(s) d s:=\mathcal{L}_{0} .
\end{aligned}
$$

For any $t \in\left(t_{i}, s_{i}\right], i=1, \ldots, N$, we have

$$
E\|(\bar{\Phi} y)(t)\|^{2} \leq c_{i}\left\|y_{t}+\tilde{\varphi}_{t}\right\|_{\mathcal{B}}^{2}+d_{i} \leq c_{i} r^{*}+d_{i}:=\mathcal{K}_{i} .
$$

Similarly, for any $t \in\left(s_{i}, t_{i+1}\right], i=1, \ldots, N$, we have

$$
\begin{aligned}
E\|(\bar{\Phi} y)(t)\|^{2} \leq & 2 M^{2}\left[c_{i}\left\|y_{s_{i}}+\tilde{\varphi}_{s_{i}}\right\|_{\mathcal{B}}^{2}+d_{i}+\left\|(-A)^{-\beta}\right\|^{2}\left(L_{1}\left\|y_{s_{i}}+\tilde{\varphi}_{s_{i}}\right\|_{\mathcal{B}}^{2}+L_{2}\right)\right] \\
& +6\left\|(-A)^{-\beta}\right\|^{2}\left(L_{1}\left\|y_{t}+\tilde{\varphi}_{t}\right\|_{\mathcal{B}}^{2}+L_{2}\right) \\
& +6 M_{1-\beta}^{2}\left(t_{i+1}-s_{i}\right) \int_{s_{i}}^{t}(t-s)^{-2(1-\beta)}\left(L_{1}\left\|y_{s}+\tilde{\varphi}_{s}\right\|_{\mathcal{B}}^{2}+L_{2}\right) d s \\
& +6 M_{1-\beta}^{2} M_{1}^{2}\left(t_{i+1}-s_{i}\right)^{2} \int_{0}^{t}(t-s)^{-2(1-\beta)} \int_{s_{i}}^{s}\left(L_{1}\left\|y_{\tau}+\tilde{\varphi}_{\tau}\right\|_{\mathcal{B}}^{2}+L_{2}\right) d \tau d s \\
& +6 M^{2}\left(t_{i+1}-s_{i}\right) \int_{s_{i}}^{t} m_{f}(s) \Theta_{f}\left(\left\|y_{s}+\tilde{\varphi}_{s}\right\|_{\mathcal{B}}^{2}\right) d s \\
& +6 M^{2} \operatorname{Tr}(Q) \int_{s_{i}}^{t} m_{F}(s) \Theta_{F}\left(\left\|y_{s}+\tilde{\varphi}_{s}\right\|_{\mathcal{B}}^{2}\right) d s \\
\leq & 12 M^{2}\left[c_{i} r^{*}+d_{i}+\left\|(-A)^{-\beta}\right\|^{2}\left(L_{1} r^{*}+L_{2}\right)\right] \\
& +6\left\|(-A)^{-\beta}\right\|^{2}\left(L_{1} r^{*}+L_{2}\right) \\
& +6 M_{1-\beta}^{2}\left(1+M_{1}^{2}\left(t_{i+1}-s_{i}\right)^{2}\right) \frac{\left(t_{i+1}-s_{i}\right)^{2 \beta}}{2 \beta-1}\left(L_{1} r^{*}+L_{2}\right) \\
& +6 M^{2}\left(t_{i+1}-s_{i}\right) \Theta_{f}\left(r^{*}\right) \int_{s_{i}}^{t_{i+1}} m_{f}(s) d s \\
& +6 M^{2} \Theta_{F}\left(r^{*}\right) \operatorname{Tr}(Q) \int_{s_{i}}^{t_{i+1}} m_{F}(s) d s:=\mathcal{L}_{i} .
\end{aligned}
$$

Take $\mathcal{L}=\max _{1 \leq i \leq N}\left\{\mathcal{L}_{0}, \mathcal{K}_{i}, \mathcal{L}_{i}\right\}$, then for each $y \in B_{r}\left(0, \mathcal{B}_{b}^{0}\right)$, we have $E\|\bar{\Phi} y\|^{2} \leq \mathcal{L}$.

Step 4. $\bar{\Phi}$ is $\chi$-contraction.

To clarify this, we decompose $\bar{\Phi}$ in the form $\bar{\Phi}=\bar{\Phi}_{1}+\bar{\Phi}_{2}$ for $t \in[0, b]$, where

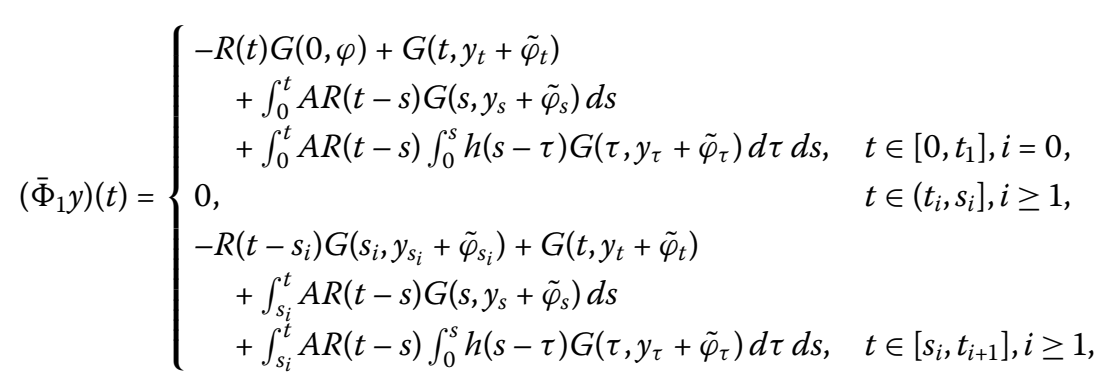




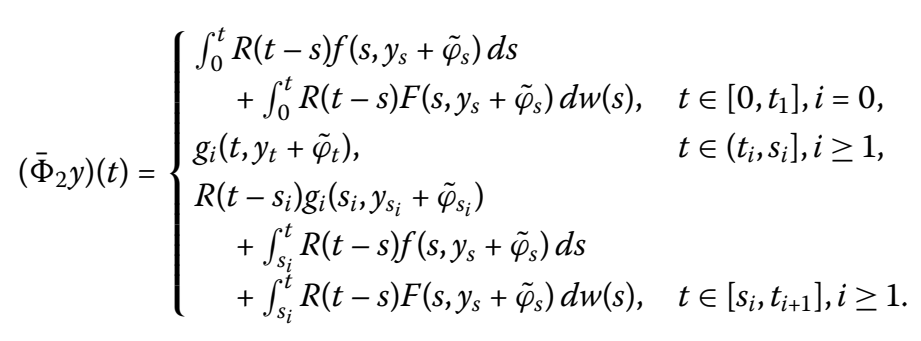

Claim $1 \bar{\Phi}_{1}$ is Lipschitz continuous.

Let $t \in\left[0, t_{1}\right]$ and $u, v \in \mathcal{B}_{b}^{0}$. From (H2) and Lemma 2.1, we have

$$
\begin{aligned}
E \|( & \left.\bar{\Phi}_{1} u\right)(t)-\left(\bar{\Phi}_{1} v\right)(t) \|^{2} \\
\leq & 3 E\left\|G\left(t, u_{t}+\tilde{\varphi}_{t}\right)-G\left(t, v_{t}+\tilde{\varphi}_{t}\right)\right\|^{2} \\
& +3 E\left\|\int_{0}^{t} A R(t-s)\left[G\left(s, u_{s}+\tilde{\varphi}_{s}\right)-G\left(s, v_{s}+\tilde{\varphi}_{s}\right)\right] d s\right\|^{2} \\
& +3 E\left\|\int_{0}^{t} A R(t-s) \int_{0}^{s} h(s-\tau)\left[G\left(\tau, u_{\tau}+\tilde{\varphi}_{\tau}\right)-G\left(\tau, v_{\tau}+\tilde{\varphi}_{\tau}\right)\right] d \tau d s\right\|^{2} \\
\leq & 3\left\|(-A)^{-\beta}\right\|^{2} L\left\|u_{t}-v_{t}\right\|_{\mathcal{B}}^{2}+3 M_{1-\beta}^{2} t_{1} \int_{0}^{t}(t-s)^{-2(1-\beta)} L\left\|u_{s}-v_{s}\right\|_{\mathcal{B}}^{2} d s \\
& +3 M_{1-\beta}^{2} M_{1}^{2} t_{1}^{2} \int_{0}^{t}(t-s)^{-2(1-\beta)} \int_{0}^{s} L\left\|u_{\tau}-v_{\tau}\right\|_{\mathcal{B}}^{2} d \tau d s \\
\leq & 12 K_{b}^{2} L\left[\left\|(-A)^{-\beta}\right\|^{2}+M_{1-\beta}^{2}\left(1+M_{1}^{2} b^{2}\right) \frac{b^{2 \beta}}{2 \beta-1}\right]\|u-v\|_{b}^{2} .
\end{aligned}
$$

Similarly, for any $t \in\left(t_{i}, t_{i+1}\right], i=1, \ldots, N$, we have

$$
\begin{aligned}
& E\left\|\left(\bar{\Phi}_{1} u\right)(t)-\left(\bar{\Phi}_{1} v\right)(t)\right\|^{2} \\
& \leq 4 E\left\|R\left(t-s_{i}\right)\left[G\left(s_{i}, u_{s_{i}}+\tilde{\varphi}_{s_{i}}\right)-G\left(s_{i}, v_{s_{i}}+\tilde{\varphi}_{s_{i}}\right)\right]\right\|^{2} \\
&+4 E\left\|G\left(t, u_{t}+\tilde{\varphi}_{t}\right)-G\left(t, v_{t}+\tilde{\varphi}_{t}\right)\right\|^{2} \\
&+4 E\left\|\int_{s_{i}}^{t} A R(t-s)\left[G\left(s, u_{s}+\tilde{\varphi}_{s}\right)-G\left(s, v_{s}+\tilde{\varphi}_{s}\right)\right] d s\right\|^{2} \\
&+4 E\left\|\int_{s_{i}}^{t} A R(t-s) \int_{0}^{s} h(s-\tau)\left[G\left(\tau, u_{\tau}+\tilde{\varphi}_{\tau}\right)-G\left(\tau, v_{\tau}+\tilde{\varphi}_{\tau}\right)\right] d \tau d s\right\|^{2} \\
& \leq 4 M^{2}\left\|(-A)^{-\beta}\right\|^{2} L\left\|u_{s_{i}}-v_{s_{i}}\right\|_{\mathcal{B}}^{2}+4\left\|(-A)^{-\beta}\right\| L\left\|u_{t}-v_{t}\right\|_{\mathcal{B}}^{2} \\
&+4 M_{1-\beta}^{2}\left(t_{i+1}-s_{i}\right) \int_{s_{i}}^{t}(t-s)^{-2(1-\beta)} L\left\|u_{s}-v_{s}\right\|_{\mathcal{B}}^{2} d s \\
&+4 M_{1-\beta}^{2} M_{1}^{2}\left(t_{i+1}-s_{i}\right)^{2} \int_{s_{i}}^{t}(t-s)^{-2(1-\beta)} \int_{0}^{s} L\left\|u_{\tau}-v_{\tau}\right\|_{\mathcal{B}}^{2} d \tau d s \\
& \leq 16 K_{b}^{2} L\left[\left(1+M^{2}\right)\left\|(-A)^{-\beta}\right\|^{2}+M_{1-\beta}^{2}\left(1+M_{1}^{2} b^{2}\right) \frac{b^{2 \beta}}{2 \beta-1}\right]\|u-v\|_{b}^{2} .
\end{aligned}
$$

Thus, for all $t \in[0, b]$, we have

$$
E\left\|\left(\bar{\Phi}_{1} u\right)(t)-\left(\bar{\Phi}_{1} v\right)(t)\right\|^{2} \leq L_{0}\|u-v\|_{b}^{2}
$$


Taking the supremum over $t$,

$$
\left\|\bar{\Phi}_{1} u-\bar{\Phi}_{1} v\right\|_{b}^{2} \leq L_{0}\|u-v\|_{b}^{2}
$$

where $L_{0}=16 K_{b}^{2} L\left[\left(1+M^{2}\right)\left\|(-A)^{-\beta}\right\|^{2}+M_{1-\beta}^{2}\left(1+M_{1}^{2} b^{2}\right) \frac{b^{2 \beta}}{2 \beta-1}\right]$. By (3.1), we see that $L_{0}<1$. Hence, $\bar{\Phi}_{1}$ is Lipschitz continuous.

Claim $2 \bar{\Phi}_{2}$ maps bounded sets into equicontinuous sets of $\mathcal{B}_{b}^{0}$.

Let $0<\tau_{1}<\tau_{2} \leq t_{1}$. For each $x \in B_{r}\left(0, \mathcal{B}_{b}^{0}\right)$, we have

$$
\begin{aligned}
& E\left\|\left(\bar{\Phi}_{2} y\right)\left(\tau_{2}\right)-\left(\bar{\Phi}_{2} y\right)\left(\tau_{1}\right)\right\|^{2} \\
& \leq 4 E\left\|\int_{0}^{\tau_{1}}\left[R\left(\tau_{2}-s\right)-R\left(\tau_{1}-s\right)\right] f\left(s, y_{s}+\tilde{\varphi}_{s}\right) d s\right\|^{2} \\
& +4 E\left\|\int_{\tau_{1}}^{\tau_{2}} R\left(\tau_{2}-s\right) f\left(s, y_{s}+\tilde{\varphi}_{s}\right) d s\right\|^{2} \\
& +4 E\left\|\int_{0}^{\tau_{1}}\left[R\left(\tau_{2}-s\right)-R\left(\tau_{1}-s\right)\right] F\left(s, y_{s}+\tilde{\varphi}_{s}\right) d w(s)\right\|^{2} \\
& +4 E\left\|\int_{\tau_{1}}^{\tau_{2}} R\left(\tau_{2}-s\right) F\left(s, y_{s}+\tilde{\varphi}_{s}\right) d w(s)\right\|^{2} \\
& \leq 4 \Theta_{f}\left(r^{*}\right) \tau_{1} \int_{0}^{\tau_{1}}\left\|R\left(\tau_{2}-s\right)-R\left(\tau_{1}-s\right)\right\|^{2} m_{f}(s) d s \\
& +4 M^{2} \Theta_{f}\left(r^{*}\right)\left(\tau_{2}-\tau_{1}\right) \int_{\tau_{1}}^{\tau_{2}} m_{f}(s) d s \\
& +4 \Theta_{F}\left(r^{*}\right) \operatorname{Tr}(Q) \int_{0}^{\tau_{1}}\left\|R\left(\tau_{2}-s\right)-R\left(\tau_{1}-s\right)\right\|^{2} m_{F}(s) d s \\
& +4 M^{2} \Theta_{F}\left(r^{*}\right) \operatorname{Tr}(Q) \int_{\tau_{1}}^{\tau_{2}} m_{F}(s) d s
\end{aligned}
$$

For any $\tau_{1}, \tau_{2} \in\left(t_{i}, s_{i}\right], \tau_{1}<\tau_{2}, i=1, \ldots, N$, we have

$$
E\left\|\left(\bar{\Phi}_{2} y\right)\left(\tau_{2}\right)-\left(\bar{\Phi}_{2} y\right)\left(\tau_{1}\right)\right\|^{2}=E\left\|g_{i}\left(\tau_{2}, y_{\tau_{2}}+\tilde{\varphi}_{\tau_{2}}\right)-g_{i}\left(\tau_{1}, y_{\tau_{1}}+\tilde{\varphi}_{\tau_{1}}\right)\right\|^{2}
$$

Similarly, for any $\tau_{1}, \tau_{2} \in\left(t_{i}, t_{i+1}\right], \tau_{1}<\tau_{2}, i=1, \ldots, N$, we have

$$
\begin{aligned}
& E\left\|\left(\bar{\Phi}_{2} y\right)\left(\tau_{2}\right)-\left(\bar{\Phi}_{2} y\right)\left(\tau_{1}\right)\right\|^{2} \\
& \leq 5 E\left\|\left[R\left(\tau_{2}-s_{i}\right)-R\left(\tau_{1}-s_{i}\right)\right] g_{i}\left(s_{i}, y_{s_{i}}+\tilde{\varphi}_{s_{i}}\right)\right\| \\
& \quad+5 E\left\|\int_{s_{i}}^{\tau_{1}}\left[R\left(\tau_{2}-s\right)-R\left(\tau_{1}-s\right)\right] f\left(s, y_{s}+\tilde{\varphi}_{s}\right) d s\right\|^{2} \\
& \quad+5 E\left\|\int_{\tau_{1}}^{\tau_{2}} R\left(\tau_{2}-s\right) f\left(s, y_{s}+\tilde{\varphi}_{s}\right) d s\right\|^{2} \\
& \quad+5 E\left\|\int_{s_{i}}^{\tau_{1}}\left[R\left(\tau_{2}-s\right)-R\left(\tau_{1}-s\right)\right] F\left(s, y_{s}+\tilde{\varphi}_{s}\right) d w(s)\right\|^{2}
\end{aligned}
$$




$$
\begin{aligned}
& +5 E\left\|\int_{\tau_{1}}^{\tau_{2}} R\left(\tau_{2}-s\right) F\left(s, y_{s}+\tilde{\varphi}_{s}\right) d w(s)\right\|^{2} \\
\leq & 5\left\|R\left(\tau_{2}-s_{i}\right)-R\left(\tau_{1}-s_{i}\right)\right\|^{2}\left[c_{i} r^{*}+d_{i}\right] \\
& +5 \Theta_{f}\left(r^{*}\right)\left(\tau_{1}-s_{i}\right) \int_{s_{i}}^{\tau_{1}}\left\|R\left(\tau_{2}-s\right)-R\left(\tau_{1}-s\right)\right\|^{2} m_{f}(s) d s \\
& +4 M^{2} \Theta_{f}\left(r^{*}\right)\left(\tau_{2}-\tau_{1}\right) \int_{\tau_{1}}^{\tau_{2}} m_{f}(s) d s \\
& +5 \Theta_{F}\left(r^{*}\right) \operatorname{Tr}(Q) \int_{s_{i}}^{\tau_{1}}\left\|R\left(\tau_{2}-s\right)-R\left(\tau_{1}-s\right)\right\|^{2} m_{F}(s) d s \\
& +5 M^{2} \Theta_{F}\left(r^{*}\right) \operatorname{Tr}(Q) \int_{\tau_{1}}^{\tau_{2}} m_{F}(s) d s .
\end{aligned}
$$

The right-hand side tends to zero as $\tau_{2} \rightarrow \tau_{1}$, since $R(t)$ is equicontinuous for $t>0 \mathrm{im}$ plies the continuity in the uniform operator topology, and in view of condition (H6). The equicontinuities for the cases $\tau_{1}<\tau_{2} \leq 0$ or $\tau_{1} \leq 0 \leq \tau_{2}$ are very simple. Hence $\bar{\Phi}_{2}$ maps $B_{r}\left(0, \mathcal{B}_{b}^{0}\right)$ into an equicontinuous family of functions.

Claim $3 \bar{\Phi}_{2}$ is a $\chi$-contraction.

Let there be an arbitrary bounded subset $W \subset \mathcal{B}_{b}^{0}$. Since the analytic resolvent operator $R(\cdot)$ is equicontinuous, $R(t-s) F\left(s, W_{s}+\tilde{\varphi}_{s}\right)$ is piecewise equicontinuous on each $\bar{J}_{i}=\left[s_{i}, t_{i+1}\right], i=0,1, \ldots, N$. For bounded sets $W, V \in \mathcal{P C}$, we can deduce the following:

$$
\begin{aligned}
& \left\|\int_{s_{i}}^{t} R(t-s) F\left(s, W_{s}+\tilde{\varphi}_{s}\right) d w(s)-\int_{s_{i}}^{t} R(t-s) F\left(s, V_{s}+\tilde{\varphi}_{s}\right) d w(s)\right\| \\
& \quad=\left(\left\|\int_{s_{i}}^{t} R(t-s)\left[F\left(s, W_{s}+\tilde{\varphi}_{s}\right)-F\left(s, V_{s}+\tilde{\varphi}_{s}\right)\right] d w(s)\right\|^{2}\right)^{\frac{1}{2}} \\
& \quad \leq M\left(\operatorname{Tr}(Q) \int_{s_{i}}^{t}\left\|F\left(s, W_{s}+\tilde{\varphi}_{s}\right)-F\left(s, V_{s}+\tilde{\varphi}_{s}\right)\right\|^{2} d s\right)^{\frac{1}{2}},
\end{aligned}
$$

and hence

$$
\chi\left(\int_{s_{i}}^{t} R(t-s) F\left(s, W_{s}+\tilde{\varphi}_{s}\right) d w(s)\right) \leq M\left(\operatorname{Tr}(Q) \int_{s_{i}}^{t}\left[\chi\left(F\left(s, W_{s}+\tilde{\varphi}_{s}\right)\right)\right]^{2} d s\right)^{\frac{1}{2}} .
$$

Then, from (H3)-(H5) and Lemmas 2.6, 2.7, we have, for $t \in\left[0, t_{1}\right]$,

$$
\begin{aligned}
\chi\left(\left(\bar{\Phi}_{2} W\right)(t)\right) \leq & \chi\left(\int_{0}^{t} R(t-s) f\left(s, W_{s}+\tilde{\varphi}_{s}\right) d s\right)+\chi\left(\int_{0}^{t} R(t-s) F\left(s, W_{s}+\tilde{\varphi}_{s}\right) d w(s)\right) \\
\leq & M \int_{0}^{t} \chi\left(f\left(s, W_{s}+\tilde{\varphi}_{s}\right)\right) d s+M\left(\operatorname{Tr}(Q) \int_{0}^{t}\left[\chi\left(F\left(s, W_{s}+\tilde{\varphi}_{s}\right)\right)\right]^{2} d s\right)^{\frac{1}{2}} \\
\leq & M \int_{0}^{t} \zeta_{f}(s) \sup _{-\infty<\theta \leq 0} \chi(W(s+\theta)+\tilde{\varphi}(s+\theta)) d s \\
& +M\left(\operatorname{Tr}(Q) \int_{0}^{t}\left[\zeta_{F}(s) \sup _{-\infty<\theta \leq 0} \chi(W(s+\theta)+\tilde{\varphi}(s+\theta))\right]^{2} d s\right)^{\frac{1}{2}}
\end{aligned}
$$




$$
\begin{aligned}
\leq & M \int_{0}^{t} \zeta_{f}(s) \sup _{0 \leq \tau \leq s} \chi(W(\tau)) d s \\
& +M\left(\operatorname{Tr}(Q) \int_{0}^{t}\left[\zeta_{F}(s) \sup _{0 \leq \tau \leq s} \chi(W(\tau))\right]^{2} d s\right)^{\frac{1}{2}} \\
\leq & M \int_{0}^{t} \zeta_{f}(s) d s \sup _{0 \leq s \leq b} \chi(W(s))+M\left(\operatorname{Tr}(Q) \int_{0}^{t} \zeta_{F}^{2}(s) d s\right)^{\frac{1}{2}} \sup _{0 \leq s \leq b} \chi(W(s)) \\
\leq & M\left[\int_{0}^{t} \zeta(s) d s+\left(\operatorname{Tr}(Q) \int_{0}^{t} \zeta_{F}^{2}(s) d s\right)^{\frac{1}{2}}\right] \chi_{\mathcal{P C}}(W) .
\end{aligned}
$$

For any $t \in\left(t_{i}, s_{i}\right], i=1, \ldots, N$, we have

$$
\begin{aligned}
\chi\left(\left(\bar{\Phi}_{2} W\right)(t)\right) & =\chi\left(g_{i}\left(t, W_{t}+\tilde{\varphi}_{t}\right)\right) \\
& \leq \gamma_{i} \sup _{-\infty<\theta \leq 0} \chi(W(t+\theta)+\tilde{\varphi}(t+\theta)) \\
& \leq \gamma_{i} \sup _{t_{i}<\tau \leq t} \chi(W(\tau)) \leq \gamma_{i} \sup _{0 \leq \tau \leq b} \chi(W(\tau)) \leq \gamma_{i} \chi_{\mathcal{P C}}(W) .
\end{aligned}
$$

Similarly, for any $t \in\left(s_{i}, t_{i+1}\right], i=1, \ldots, N$, we have

$$
\begin{aligned}
& \chi\left(\left(\bar{\Phi}_{2} W\right)(t)\right) \leq \chi\left(R\left(t-s_{i}\right) g_{i}\left(s_{i}, W_{s_{i}}+\tilde{\varphi}_{s_{i}}\right)\right)+\chi\left(\int_{s_{i}}^{t} R(t-s) f\left(s, W_{s}+\tilde{\varphi}_{s}\right) d s\right) \\
& +\chi\left(\int_{s_{i}}^{t} R(t-s) F\left(s, W_{s}+\tilde{\varphi}_{s}\right) d w(s)\right) \\
& \leq M \chi\left(g_{i}\left(s_{i}, W_{s_{i}}+\tilde{\varphi}_{s_{i}}\right)\right)+M \int_{s_{i}}^{t} \chi\left(f\left(s, W_{s}+\tilde{\varphi}_{s}\right)\right) d s \\
& +M\left(\operatorname{Tr}(Q) \int_{s_{i}}^{t}\left[\chi\left(F\left(s, W_{s}+\tilde{\varphi}_{s}\right)\right)\right]^{2} d s\right)^{\frac{1}{2}} \\
& \leq M \gamma_{i} \sup _{-\infty<\theta \leq 0} \chi\left(W\left(s_{i}+\theta\right)+\tilde{\varphi}\left(s_{i}+\theta\right)\right) \\
& +M \int_{s_{i}}^{t} \zeta_{f}(s) \sup _{-\infty<\theta \leq 0} \chi(W(s+\theta)+\tilde{\varphi}(s+\theta)) d s \\
& +M\left(\operatorname{Tr}(Q) \int_{s_{i}}^{t}\left[\zeta_{F}(s) \sup _{-\infty<\theta \leq 0} \chi(W(s+\theta)+\tilde{\varphi}(s+\theta))\right]^{2} d s\right)^{\frac{1}{2}} \\
& \leq M \gamma_{i} \sup _{s_{i}<\tau \leq t} \chi(W(\tau))+M \int_{s_{i}}^{t} \zeta_{f}(s) \sup _{s_{i}<\tau \leq s} \chi(W(\tau)) d s \\
& +M\left(\operatorname{Tr}(Q) \int_{s_{i}}^{t}\left[\zeta_{F}(s) \sup _{s_{i} \leq \tau \leq s} \chi(W(\tau))\right]^{2} d s\right)^{\frac{1}{2}} \\
& \leq M \gamma_{i} \sup _{0 \leq \tau \leq b} \chi(W(\tau))+M \int_{s_{i}}^{t} \zeta_{f}(s) d s \sup _{0 \leq s \leq b} \chi(W(s)) \\
& +M\left(\operatorname{Tr}(Q) \int_{s_{i}}^{t} \zeta_{F}^{2}(s) d s\right)^{\frac{1}{2}} \sup _{0 \leq s \leq b} \chi(W(s)) \\
& \leq M\left[\gamma_{i}+\int_{s_{i}}^{t} \zeta_{f}(s) d s+\left(\operatorname{Tr}(Q) \int_{s_{i}}^{t} \zeta_{F}^{2}(s) d s\right)^{\frac{1}{2}}\right] \chi_{\mathcal{P C}}(W) .
\end{aligned}
$$


Thus, for all $t \in[0, b]$, we have

$$
\chi\left(\left(\bar{\Phi}_{2} W\right)(t)\right) \leq\left[(M+1) \gamma_{i}+M \int_{0}^{b} \zeta_{f}(s) d s+M\left(\operatorname{Tr}(Q) \int_{0}^{t} \zeta_{F}^{2}(s) d s\right)^{\frac{1}{2}}\right] \chi_{\mathcal{P C}}(W)
$$

and

$$
\chi\left(\bar{\Phi}_{2} W\right) \leq\left[(M+1) \gamma_{i}+M \int_{0}^{b} \zeta_{f}(s) d s+M\left(\operatorname{Tr}(Q) \int_{0}^{b} \zeta_{F}^{2}(s) d s\right)^{\frac{1}{2}}\right] \chi_{\mathcal{P C}}(W)
$$

Since

$$
\begin{aligned}
\chi_{\mathcal{P C}}(\bar{\Phi} W) & =\chi_{\mathcal{P C}}\left(\bar{\Phi}_{1} W+\bar{\Phi}_{2} W\right) \leq \chi_{\mathcal{P C}}\left(\bar{\Phi}_{1} W\right)+\chi_{\mathcal{P C}}\left(\bar{\Phi}_{2} W\right) \\
& \leq \tilde{L} \chi_{\mathcal{P C}}(W)<\chi_{\mathcal{P C}}(W)
\end{aligned}
$$

where $\tilde{L}=\max _{1 \leq i \leq N}\left\{\sqrt{L_{0}}+(M+1) \gamma_{i}+M \int_{0}^{b} \zeta_{f}(s) d s+M\left(\operatorname{Tr}(Q) \int_{0}^{b} \zeta_{F}^{2}(s) d s\right)^{\frac{1}{2}}\right\}<1$. Therefore, $\bar{\Phi}$ is a $\chi$-contraction. In view of Lemma 2.8 , we conclude that $\bar{\Phi}$ has at least one fixed point $y^{*} \in W \subset \mathcal{B}_{b}^{0}$. Let $x(t)=y^{*}(t)+\tilde{\varphi}(t), t \in(-\infty, b]$. Then $x$ is a fixed point of the operator $\Phi$, which implies that $x$ is a mild solution of the problem (1.1)-(1.3) and the proof of Theorem 3.1 is complete.

Theorem 3.2 If the assumptions (H1)-(H6) are satisfied, then the system (1.1)-(1.3) has at least one mild solution on $[0, b]$, provided that

$$
\begin{aligned}
& \max _{1 \leq i \leq N} 4 K_{b}^{2}\left\{\left(1+12 M^{2}\right) c_{i}+\left[\left(1+12 M^{2}\right)\left\|(-A)^{-\beta}\right\|^{2}\right.\right. \\
& \left.+6 M_{1-\beta}^{2}\left(1+M_{1}^{2} b^{2}\right) \frac{b^{2 \beta}}{2 \beta-1}\right] L_{1}+6 M^{2} b \int_{0}^{b} m_{f}(s) d s \limsup _{\varsigma \rightarrow \infty} \frac{\Theta_{f}(\varsigma)}{\varsigma} \\
& \left.+6 M^{2} \operatorname{Tr}(Q) \int_{0}^{b} m_{F}(s) d s \limsup _{\varsigma \rightarrow \infty} \frac{\Theta_{F}(\varsigma)}{\varsigma}\right\}<1 .
\end{aligned}
$$

Proof Let $\Phi, \bar{\Phi}$ be defined as in the proof of Theorem 3.1. We claim that there exists an $k>0$ such that $\bar{\Phi}\left(B_{k}\left(0, \mathcal{B}_{b}^{0}\right)\right) \subset\left(B_{k}\left(0, \mathcal{B}_{b}^{0}\right)\right)$. If we assume that this assertion is false, then for each $k>0$ we can choose $y^{k} \in B_{k}\left(0, \mathcal{B}_{b}^{0}\right)$ and $t^{k} \in[0, b]$ such that $k<E\left\|\left(\Phi y^{k}\right)\left(t^{k}\right)\right\|^{2}$. Consequently, we have, for $t^{k} \in\left[0, t_{1}\right]$,

$$
\begin{aligned}
k< & E\left\|\left(\Phi y^{k}\right)\left(t^{k}\right)\right\|^{2} \\
\leq & 6 M\left\|(-A)^{-\beta}\right\|^{2}\left(L_{1}\|\varphi\|_{\mathcal{B}}^{2}+L_{2}\right) \\
& +6\left\|(-A)^{-\beta}\right\|^{2}\left(L_{1}\left\|y_{t^{k}}^{k}+\tilde{\varphi}_{t^{k}}\right\|_{\mathcal{B}}^{2}+L_{2}\right) \\
& +6 M_{1-\beta}^{2} t_{1} \int_{0}^{t^{k}}\left(t^{k}-s\right)^{-2(1-\beta)}\left(L_{1}\left\|y_{s}^{k}+\tilde{\varphi}_{s}\right\|_{\mathcal{B}}^{2}+L_{2}\right) d s \\
& +6 M_{1-\beta}^{2} M_{1} t_{1}^{2} \int_{0}^{t^{k}}\left(t^{k}-s\right)^{-2(1-\beta)} \int_{0}^{s}\left(L_{1}\left\|y_{\tau}^{k}+\tilde{\varphi}_{\tau}\right\|_{\mathcal{B}}^{2}+L_{2}\right) d \tau d s \\
& +6 M^{2} t_{1} \int_{0}^{t^{k}} m_{f}(s) \Theta_{f}\left(\left\|y_{s}^{k}+\tilde{\varphi}_{s}\right\|_{\mathcal{B}}^{2}\right) d s+6 M^{2} \operatorname{Tr}(Q) \int_{0}^{t^{k}} m_{F}(s) \Theta_{F}\left(\left\|y_{s}^{k}+\tilde{\varphi}_{s}\right\|_{\mathcal{B}}^{2}\right) d s .
\end{aligned}
$$


For any $t^{k} \in\left(t_{i}, s_{i}\right], i=1, \ldots, N$, we have

$$
k<E\left\|\left(\Phi y^{k}\right)\left(t^{k}\right)\right\|^{2} \leq c_{i}\left\|y_{t^{k}}^{k}+\tilde{\varphi}_{t^{k}}\right\|_{\mathcal{B}}^{2}+d_{i}
$$

Similarly, for any $t^{k} \in\left(s_{i}, t_{i+1}\right], i=1, \ldots, N$, we have

$$
\begin{aligned}
k< & E\left\|\left(\Phi y^{k}\right)\left(t^{k}\right)\right\|^{2} \\
\leq & 12 M\left[c_{i}\left\|y_{s_{i}}^{k}+\tilde{\varphi}_{s_{i}}\right\|_{\mathcal{B}}^{2}+d_{i}+\left\|(-A)^{-\beta}\right\|^{2}\left(L_{1}\left\|y_{s_{i}}^{k}+\tilde{\varphi}_{s_{i}}\right\|_{\mathcal{B}}^{2}+L_{2}\right)\right] \\
& +6\left\|(-A)^{-\beta}\right\|^{2}\left(L_{1}\left\|y_{t^{k}}^{k}+\tilde{\varphi}_{t^{k}}\right\|_{\mathcal{B}}^{2}+L_{2}\right) \\
& +6 M_{1-\beta}^{2}\left(t_{i+1}-s_{i}\right) \int_{s_{i}}^{t^{k}}\left(t^{k}-s\right)^{-2(1-\beta)}\left(L_{1}\left\|y_{s}^{k}+\tilde{\varphi}_{s}\right\|_{\mathcal{B}}^{2}+L_{2}\right) d s \\
& +6 M_{1-\beta}^{2} M_{1}^{2}\left(t_{i+1}-s_{i}\right)^{2} \int_{s_{i}}^{t^{k}}\left(t^{k}-s\right)^{-2(1-\beta)} \int_{0}^{s}\left(L_{1}\left\|y_{\tau}^{k}+\tilde{\varphi}_{\tau}\right\|_{\mathcal{B}}^{2}+L_{2}\right) d \tau d s \\
& +6 M^{2}\left(t_{i+1}-s_{i}\right) \int_{s_{i}}^{t^{k}} m_{f}(s) \Theta_{f}\left(\left\|y_{s}^{k}+\tilde{\varphi}_{s}\right\|_{\mathcal{B}}^{2}\right) d s \\
& +6 M^{2} \operatorname{Tr}(Q) \int_{s_{i}}^{t^{k}} m_{F}(s) \Theta_{F}\left(\left\|y_{s}^{k}+\tilde{\varphi}_{s}\right\|_{\mathcal{B}}^{2}\right) d s .
\end{aligned}
$$

Then, for all $t^{k} \in[0, b]$, we have

$$
\begin{aligned}
k< & E\left\|\left(\Phi y^{k}\right)\left(t^{k}\right)\right\|^{2} \\
\leq & M^{*}+4 c_{i}\left[K_{b}^{2} k+\left(K_{b}^{2} M^{2} \tilde{H}^{2}+M_{b}^{2}\right)\|\varphi\|_{\mathcal{B}}^{2}\right]+12 M^{2}\left\{4 c _ { i } \left[K_{b}^{2} k\right.\right. \\
& \left.\left.+\left(K_{b}^{2} M^{2} \tilde{H}^{2}+M_{b}^{2}\right)\|\varphi\|_{\mathcal{B}}^{2}\right]+\left\|(-A)^{-\beta}\right\|^{2} L_{1} 4\left[K_{b}^{2} k+\left(K_{b}^{2} M^{2} \tilde{H}^{2}+M_{b}^{2}\right)\|\varphi\|_{\mathcal{B}}^{2}\right]\right\} \\
& +6\left\|(-A)^{-\beta}\right\|^{2} L_{1} 4\left[K_{b}^{2} k+\left(K_{b}^{2} M^{2} \tilde{H}^{2}+M_{b}^{2}\right)\|\varphi\|_{\mathcal{B}}^{2}\right] \\
& +6 M_{1-\beta}^{2} b \int_{0}^{t^{k}}\left(t^{k}-s\right)^{-2(1-\beta)} L_{1} 4\left[K_{b}^{2} k+\left(K_{b}^{2} M^{2} \tilde{H}^{2}+M_{b}^{2}\right)\|\varphi\|_{\mathcal{B}}^{2}\right] d s \\
& +6 M_{1-\beta}^{2} M_{1}^{2} b^{2} \int_{0}^{t^{k}}\left(t^{k}-s\right)^{-2(1-\beta)} \int_{0}^{s} L_{1} 4\left[K_{b}^{2} k+\left(K_{b}^{2} M^{2} \tilde{H}^{2}+M_{b}^{2}\right)\|\varphi\|_{\mathcal{B}}^{2}\right] d \tau d s \\
& +6 M b \int_{0}^{t^{k}} m_{f}(s) \Theta_{f}\left(4\left[K_{b}^{2} k+\left(K_{b}^{2} M^{2} \tilde{H}^{2}+M_{b}^{2}\right)\|\varphi\|_{\mathcal{B}}^{2}\right]\right) d s \\
& +6 M^{2} \operatorname{Tr}(Q) \int_{0}^{t^{k}} m_{F}(s) \Theta_{F}\left(4\left[K_{b}^{2} k+\left(K_{b}^{2} M^{2} \tilde{H}^{2}+M_{b}^{2}\right)\|\varphi\|_{\mathcal{B}}^{2}\right]\right) d s,
\end{aligned}
$$

and hence

$$
\begin{aligned}
1< & \max _{1 \leq i \leq N} 4 K_{b}^{2}\left\{\left(1+12 M^{2}\right) c_{i}+\left[\left(1+12 M^{2}\right)\left\|(-A)^{-\beta}\right\|^{2}\right.\right. \\
& \left.+6 M_{1-\beta}^{2}\left(1+M_{1}^{2} b^{2}\right) \frac{b^{2 \beta}}{2 \beta-1}\right] L_{1}+6 M^{2} b \int_{0}^{b} m_{f}(s) d s \limsup _{\varsigma \rightarrow \infty} \frac{\Theta_{f}(\varsigma)}{\varsigma} \\
& \left.+6 M^{2} \operatorname{Tr}(Q) \int_{0}^{b} m_{F}(s) d s \limsup _{\varsigma \rightarrow \infty} \frac{\Theta_{F}(\varsigma)}{\varsigma}\right\},
\end{aligned}
$$


which is contrary to our assumption (3.6). The proofs of the other steps are similar to those in Theorem 3.1. Therefore, we omit the details. By means of Lemma 2.9, we conclude that (1.1)-(1.3) has at least a mild solution. The proof is complete.

\section{Application}

Consider the impulsive stochastic partial functional neutral integro-differential equations of the form

$$
\begin{aligned}
& d\left[z(t, x)-\int_{-\infty}^{t} \vartheta_{1}(t) \vartheta_{2}(s-t) z(s, x) d s\right] \\
& =\frac{\partial^{2}}{\partial x^{2}}\left[z(t, x)+\int_{0}^{t} \tilde{b}(t-s) z(s, x) d s\right] d t \\
& \quad+\int_{-\infty}^{t} v_{1}(t) v_{1}(s-t) z(s, x) d s d t \\
& \quad+\int_{-\infty}^{t} \varpi_{1}(t) \varpi_{1}(s-t) z(s, x) d s d w(t), \\
& \quad(t, x) \in \bigcup_{i=1}^{N}\left[s_{i}, t_{i+1}\right] \times[0, \pi], \\
& z(t, 0)=z(t, \pi)=0, \quad t \in[0, b], \\
& z(\tau, x)=\varphi(\tau, x), \quad(\tau, x) \in(-\infty, 0] \times \in[0, \pi], \\
& z(t, x)=\int_{-\infty}^{t} \eta_{i}(s-t) z(s, x) d s, \quad(t, x) \in\left(t_{i}, s_{i}\right] \times[0, \pi],
\end{aligned}
$$

where $\varphi$ is continuous and there exists a constant $K_{1}$ such that $|\tilde{b}(t-s)| \leq K_{1}$. w( $(t)$ denotes a one-dimensional standard Wiener process in $H$ defined on a stochastic space $(\Omega, \mathcal{F}, P)$ and we take $H=L^{2}([0,1])$ with the norm $\|\cdot\|$ and define the operators $A: H \rightarrow H$ by $A \omega=\omega^{\prime \prime}$ with the domain

$$
D(A):=\left\{\omega \in H: \omega, \omega^{\prime} \text { are absolutely continuous, } \omega^{\prime \prime} \in H, \omega(0)=\omega(\pi)=0\right\} .
$$

Then

$$
A \omega=-\sum_{n=1}^{\infty} n^{2}\left\langle\omega, z_{n}\right\rangle z_{n}, \quad \omega \in D(A)
$$

where $z_{n}(x)=\sqrt{\frac{2}{\pi}} \sin (n x), n=1,2,3, \ldots$, is an orthogonal set of eigenvector of $-A$. It is well known that $A$ generates a strongly continuous semigroup that is analytic, and a resolvent operator $R(t)$ can be extracted from this analytic semigroup (see [27, 28]). Since the operator $R(t)$ is analytic there exists a constant $M>0$ such that $\|R(t)\| \leq M$ and it satisfies (H1).

The bounded linear operator $(-A)^{\frac{3}{4}}$ is given by

$$
(-A)^{\frac{3}{4}} \omega=\sum_{n=1}^{\infty} n^{\frac{3}{2}}\left\langle\omega, z_{n}\right\rangle z_{n}
$$


on the space

$$
D\left((-A)^{\frac{3}{4}}\right)=\left\{\omega(\cdot) \in H: \sum_{n=1}^{\infty} n^{\frac{3}{2}}\left\langle\omega, z_{n}\right\rangle z_{n} \in H\right\},
$$

and $(-A)^{-\frac{3}{4}} \omega=\sum_{n=1}^{\infty} \frac{1}{n^{\frac{3}{2}}}\left\langle\omega, z_{n}\right\rangle z_{n}$ for every $\omega \in H$ and $\left\|(-A)^{-\frac{3}{4}}\right\|$ is bounded.

Let $r \geq 0,1 \leq p<+\infty$ and let $\tilde{h}:(-\infty,-r] \rightarrow \mathbb{R}$ be a nonnegative measurable function which satisfies the conditions (h-5), (h-6) in the terminology of Hino et al. [36]. Briefly, this means that $\tilde{h}$ is locally integrable and there is a nonnegative, locally bounded function $\gamma$ on $(-\infty, 0]$ such that $\tilde{h}(\xi+\tau) \leq \gamma(\xi) \tilde{h}(\tau)$ for all $\xi \leq 0$ and $\theta \in(-\infty,-r) \backslash N_{\xi}$, where $N_{\xi} \subseteq(-\infty,-r)$ is a set whose Lebesgue measure zero. We denote by $\mathcal{P} \mathcal{C}_{r} \times L^{p}(\tilde{h}, H)$ the set that consists of all classes of functions $\varphi:(-\infty, 0] \rightarrow H$ such that $\varphi_{[-r, 0]} \in \mathcal{P C}([-r, 0], H)$, $\varphi(\cdot)$ is Lebesgue measurable on $(-\infty,-r)$, and $\tilde{h}\|\varphi\|^{p}$ is Lebesgue integrable on $(-\infty,-r)$. The seminorm is given by

$$
\|\varphi\|_{\mathcal{B}}=\sup _{-r \leq \tau \leq 0}\|\varphi(\tau)\|+\left(\int_{-\infty}^{-r} \tilde{h}(\tau)\|\varphi\|^{p} d \tau\right)^{1 / p} .
$$

The space $\mathcal{B}=\mathcal{P C}_{r} \times L^{p}(\tilde{h}, H)$ satisfies axioms (A)-(C). Moreover, when $r=0$ and $p=2$, we can take $\tilde{H}=1, M(t)=\gamma(-t)^{1 / 2}$, and $K(t)=1+\left(\int_{-t}^{0} \tilde{h}(\tau) d \tau\right)^{1 / 2}$, for $t \geq 0$. (See Theorem 1.3.8 in [36] for details.)

Additionally, assume that the following conditions hold:

(i) The functions $\vartheta_{j}, v_{j}, \varpi_{j}: \mathbb{R} \rightarrow \mathbb{R}, j=1,2$, are continuous with

$$
L_{\vartheta_{2}}=\int_{-\infty}^{0} \frac{\left(\vartheta_{2}(s)\right)^{2}}{\tilde{h}(s)} d s<\infty, L_{v_{2}}=\int_{-\infty}^{0} \frac{\left(v_{2}(s)\right)^{2}}{\tilde{h}(s)} d s<\infty, L_{\varpi_{2}}=\int_{-\infty}^{0} \frac{\left(\varpi_{2}(s)\right)^{2}}{\tilde{h}(s)} d s<\infty .
$$

(ii) The functions $\eta_{i}: \mathbb{R} \rightarrow \mathbb{R}, i=1, \ldots, N$, are continuous with $\tilde{L}_{i}=\int_{-\infty}^{0} \frac{\left(\eta_{i}(s)\right)^{2}}{\tilde{h}(s)} d s<\infty$ for every $i=1,2, \ldots, N$.

Take $\varphi \in \mathcal{B}=\mathcal{P C}_{0} \times L^{2}(\tilde{h}, H)$ with $\varphi(\theta)(x)=\varphi(\theta, x),(\theta, x) \in(-\infty, 0] \times \mathcal{B}$. Let $z(s)(x)=$ $z(s, x) . G:[0, b] \times \mathcal{B} \rightarrow H, f:[0, b] \times \mathcal{B} \rightarrow H, F:[0, b] \times \mathcal{B} \rightarrow L(H), g_{i}:[0, b] \times \mathcal{B} \rightarrow H$ be the operators defined by

$$
\begin{aligned}
& (-A)^{\frac{3}{4}} G(t, \varphi)(x)=\int_{-\infty}^{0} \vartheta_{1}(t) \vartheta_{2}(\theta) \varphi(\theta)(x) d \theta, \\
& f(t, \varphi)(x)=\int_{-\infty}^{0} v_{1}(t) \mu_{2}(\theta) \varphi(\theta)(x) d \theta \\
& F(t, \varphi)(x)=\int_{-\infty}^{0} \varpi_{1}(t) \varpi_{2}(\theta) \varphi(\theta)(x) d \theta \\
& g_{i}(t, \varphi)(x)=\int_{-\infty}^{0} \eta_{i}(\theta) \varphi(\theta)(x) d \theta .
\end{aligned}
$$

Under the above assumptions, the problem (4.1)-(4.4) can be written as (1.1)-(1.3). Moreover, for any $t \in[0, b], \psi, \psi_{1} \in \mathcal{B}$, we have

$$
\begin{aligned}
E\left\|(-A)^{\frac{3}{4}} G(t, \psi)\right\|^{2} & =E\left[\int_{0}^{\pi}\left(\int_{-\infty}^{0} \vartheta_{1}(t) \vartheta_{2}(s) \psi(s)(x) d s\right)^{2} d x\right] \\
& \leq\left(\int_{-\infty}^{0} \vartheta_{1}(t) \vartheta_{2}(s)\|\psi(s)\| d s\right)^{2}
\end{aligned}
$$




$$
\begin{aligned}
& \leq\left\|\vartheta_{1}\right\|_{\infty}^{2}\left(\int_{-\infty}^{0} \frac{\left(\vartheta_{2}(s)\right)^{2}}{\tilde{h}(s)} d s\right)\left(\int_{-\infty}^{0} \tilde{h}(s)\|\psi(s)\|^{2} d s\right) \\
& \leq L_{G}\|\psi\|_{\mathcal{B}}^{2}
\end{aligned}
$$

and

$$
\begin{aligned}
E \| & (-A)^{\frac{3}{4}}\left[G(t, \psi)-G\left(t, \psi_{1}\right)\right] \|^{2} \\
& =E\left[\int_{0}^{\pi}\left(\int_{-\infty}^{0} \vartheta_{1}(t) \vartheta_{2}(s)\left[\psi(s)(x)-\psi_{1}(s)(x)\right] d s\right)^{2} d x\right] \\
& \leq\left(\int_{-\infty}^{0} \vartheta_{1}(t) \vartheta_{2}(s)\left\|\psi(s)-\psi_{1}(s)\right\| d s\right)^{2} \\
& \leq\left\|\vartheta_{1}\right\|_{\infty}^{2}\left(\int_{-\infty}^{0} \frac{\left(\vartheta_{2}(s)\right)^{2}}{\tilde{h}(s)} d s\right)\left(\int_{-\infty}^{0} \tilde{h}(s)\left\|\psi(s)-\psi_{1}(s)\right\|^{2} d s\right) \\
& \leq L_{G}\left\|\psi-\psi_{1}\right\|_{\mathcal{B}}^{2},
\end{aligned}
$$

where $L_{G}=\left\|\vartheta_{1}\right\|_{\infty}^{2} L_{\vartheta_{2}}$. For any $t \in[0, b], \psi, \psi_{1} \in \mathcal{B}$, we have

$$
\begin{aligned}
E\|f(t, \psi)\|^{2} & =E\left[\int_{0}^{\pi}\left(\int_{-\infty}^{0} v_{1}(t) v_{2}(s) \psi(s)(x) d s\right)^{2} d x\right] \\
& \leq\left(\int_{-\infty}^{0} v_{1}(t) v_{2}(s)\|\psi(s)\| d s\right)^{2} \\
& \leq\left\|v_{1}\right\|_{\infty}^{2}\left(\int_{-\infty}^{0} \frac{\left(v_{2}(s)\right)^{2}}{\tilde{h}(s)} d s\right)\left(\int_{-\infty}^{0} \tilde{h}(s)\|\psi(s)\|^{2} d s\right) \\
& \leq L_{f}\|\psi\|_{\mathcal{B}}^{2}
\end{aligned}
$$

and

$$
\begin{aligned}
& \left\|f(t, \psi)-f\left(t, \psi_{1}\right)\right\| \\
& \quad=\left[\int_{0}^{\pi}\left(\int_{-\infty}^{0} v_{1}(t) v_{2}(s)\left[\psi(s)(x)-\psi_{1}(s)(x)\right] d s\right)^{2} d x\right]^{\frac{1}{2}} \\
& \quad \leq \int_{-\infty}^{0} v_{1}(t) v_{2}(s)\left\|\psi(s)-\psi_{1}(s)\right\| d s \\
& \leq\left\|v_{1}\right\|_{\infty}\left(\int_{-\infty}^{0} \frac{\left(v_{2}(s)\right)^{2}}{\tilde{h}(s)} d s\right)^{\frac{1}{2}}\left(\int_{-\infty}^{0} \tilde{h}(s)\left\|\psi(s)-\psi_{1}(s)\right\|^{2} d s\right)^{\frac{1}{2}} \\
& \leq \sqrt{L_{f}}\left\|\psi-\psi_{1}\right\|_{\mathcal{B}},
\end{aligned}
$$

and we can see that, for each bounded set $D \subset \mathcal{B}$,

$$
\chi(f(t, D)) \leq \sqrt{L_{f}} \sup _{-\infty<\theta \leq 0} \chi(D(\theta))
$$

where $L_{f}=\left\|v_{1}\right\|_{\infty}^{2} L_{v_{2}}$. Similarly, we have

$$
E\|F(t, \psi)\|^{2} \leq L_{F}\|\psi\|_{\mathcal{B}}^{2}
$$


and

$$
\left\|F(t, \psi)-F\left(t, \psi_{1}\right)\right\| \leq \sqrt{L_{F}}\left\|\psi-\psi_{1}\right\|_{\mathcal{B}}
$$

and we can see that, for each bounded set $D \subset \mathcal{B}$,

$$
\chi(F(t, D)) \leq \sqrt{L_{F}} \sup _{-\infty<\theta \leq 0} \chi(D(\theta))
$$

where $L_{F}=\left\|\varpi_{1}\right\|_{\infty}^{2} L_{\varpi_{2}}$.

Moreover, we have, for any $t, \tilde{t} \in\left(t_{i}, s_{i}\right], i=1, \ldots, N, \psi, \psi_{1} \in \mathcal{B}$,

$$
\begin{aligned}
& E\left\|g_{i}(t, \psi)\right\|^{2} \leq \tilde{L}_{i}\|\psi\|_{\mathcal{B}}, \\
& \left\|g_{i}(t, \psi)-g_{i}\left(\tilde{t}, \psi_{1}\right)\right\| \leq \sqrt{\tilde{L}_{i}}\left\|\psi-\psi_{1}\right\|_{\mathcal{B}},
\end{aligned}
$$

and we can see that, for each bounded set $D \subset \mathcal{B}$,

$$
\chi\left(g_{i}(t, D)\right) \leq \sqrt{\tilde{L}_{i}} \sup _{-\infty<\theta \leq 0} \chi(D(\theta))
$$

Obviously, $\Theta_{f}(s)=\Theta_{F}(s)=s, \int_{1}^{\infty} \frac{1}{\Theta_{f}(s)+\Theta_{F}(s)} d s=\infty$. If also the associated conditions (3.1) and (3.3) or (3.6) hold, then it satisfies all the assumptions given in Theorem 3.1 or Theorem 3.2, and we can conclude that the problem (4.1)-(4.4) has at least one mild solution on $[0, b]$.

\section{Conclusion}

In this paper, we studied a new class of impulsive stochastic partial neutral evolution equations with infinite delay in Hilbert spaces. More precisely, by using stochastic analysis, the theory of analytic resolvent operators, fractional power of closed operators, the Darbo fixed point theorem, and the Darbo-Sadovskii fixed point theorem combined with the Hausdorff measure of noncompactness techniques, we investigated the existence of mild solutions of the impulsive stochastic system. The conditions are formulated and proved under which $R(\cdot)$ is analytic and $f, F$ satisfy the Carathéodory condition. Finally, an application is provided to illustrate the applicability of the new results.

\section{Competing interests}

The authors declare that they have no competing interests.

\section{Authors' contributions}

The authors completed the paper, and they read and approved the final manuscript.

\section{Acknowledgements}

The authors wish to thank the anonymous referee for the kind comments. This work is supported by the National Natural Science Foundation of China (11461019), the President Fund of Scientific Research Innovation and Application of Hexi University (xz2013-10, XZ2014-22), the Scientific Research Project of Universities of Gansu Province (2014A-110).

Received: 16 November 2015 Accepted: 8 February 2016 Published online: 22 February 2016

\section{References}

1. Benchohra, M, Henderson, J, Ntouyas, SK: Impulsive Differential Equations and Inclusions. Hindawi Publishing Corporation, New York (2006)

2. Samoilenko, AM, Perestyuk, NA: Impulsive Differential Equations. World Scientific, Singapore (1995) 
3. Hernández, E, Rabello, M, Henríquez, H: Existence of solutions for impulsive partial neutral functional differential equations. J. Math. Anal. Appl. 331, 1135-1158 (2007)

4. Park, JY, Balachandran, $K$, Annapoorani, N: Existence results for impulsive neutral functional integrodifferential equations with infinite delay. Nonlinear Anal. 71, 3152-3162 (2009)

5. Mao, X: Stochastic Differential Equations and Their Applications. Horwood, Chichester (1997)

6. Sakthivel, R, Revathi, P, Anthoni, SM: Existence of pseudo almost automorphic mild solutions to stochastic fractional differential equations. Nonlinear Anal. 75, 3339-3347 (2012)

7. Ren, Y, Cheng, X, Sakthivel, R: On time-dependent stochastic evolution equations driven by fractional Brownian motion in a Hilbert space with finite delay. Math. Methods Appl. Sci. 37, 2177-2184 (2014)

8. Revathi, $P$, Sakthivel, $R$, Ren, $Y$, Anthoni, SM: Existence of almost automorphic mild solutions to non-autonomous neutral stochastic differential equations. Appl. Math. Comput. 230, 639-649 (2014)

9. Balasubramaniam, P, Tamilalagan, P: Approximate controllability of a class of fractional neutral stochastic integro-differential inclusions with infinite delay by using Mainardis function. Appl. Math. Comput. 256, 232-246 (2015)

10. Sakthivel, R, Luo, J: Asymptotic stability of impulsive stochastic partial differential equations with infinite delays. J. Math. Anal. Appl. 356, 1-6 (2009)

11. Anguraj, A, Vinodkumar, A: Existence, uniqueness and stability results of impulsive stochastic semilinear neutral functional differential equations with infinite delays. Electron. J. Qual. Theory Differ. Equ. 2009, 67 (2009)

12. $\mathrm{Hu}, \mathrm{L}, \mathrm{Ren}, \mathrm{Y}$ : Existence results for impulsive neutral stochastic functional integro-differential equations with infinite delays. Acta Appl. Math. 111, 303-317 (2010)

13. Lin, $A, R e n, Y, X i a, N$ : On neutral impulsive stochastic integro-differential equations with infinite delays via fractional operators. Math. Comput. Model. 51, 413-424 (2010)

14. Yan, Z, Yan, X: Existence of solutions for impulsive partial stochastic neutral integrodifferential equations with state-dependent delay. Collect. Math. 64, 235-250 (2013)

15. Sakthivel, R, Revathi, $P$, Ren, Y: Existence of solutions for nonlinear fractional stochastic differential equations. Nonlinear Anal. 81, 70-86 (2013)

16. Yan, Z, Zhang, H: Asymptotic stability of fractional impulsive neutral stochastic partial integro-differential equations with state-dependent delay. Electron. J. Differ. Equ. 2013, 206 (2013)

17. Balasubramaniam, P, Kumaresan, N, Ratnavelu, K, Tamilalagan, P: Local and global existence of mild solution for impulsive fractional stochastic differential equations. Bull. Malays. Math. Sci. Soc. 38, 867-884 (2015)

18. Ren, $Y$, Chenga, $X$, Sakthivel, R: Impulsive neutral stochastic functional integro-differential equations with infinite delay driven by fBm. Appl. Math. Comput. 247, 205-212 (2014)

19. Hernández, E, O'Regan, D: On a new class of abstract impulsive differential equations. Proc. Am. Math. Soc. 141, 1641-1649 (2013)

20. Pierri, M, O'Regan, D, Rolnik, V: Existence of solutions for semi-linear abstract differential equations with not instantaneous impulses. Appl. Math. Comput. 219, 6743-6749 (2013)

21. Gautam, GR, Dabas, J: Mild solution for fractional functional integro-differential equation with not instantaneous impulse. Malaya J. Mat. 2, 428-437 (2014)

22. $Y u, X$, Wang, J: Periodic boundary value problems for nonlinear impulsive evolution equations on Banach spaces. Commun. Nonlinear Sci. Numer. Simul. 22, 980-989 (2015)

23. Fu, X, Liu, X, Lu, B: On a new class of impulsive fractional evolution equations. Adv. Differ. Equ. 2015, 227 (2015)

24. Yan, Z, Lu, F: Existence results for a new class of fractional impulsive partial neutral stochastic integro-differential equations with infinite delay. J. Appl. Anal. Comput. 5, 329-346 (2015)

25. Hernández, E, O'Regan, D: Controllability of Volterra-Fredholm type systems in Banach spaces. J. Franklin Inst. 346 95-101 (2009)

26. Obukhovski, V, Zecca, P: Controllability for systems governed by semilinear differential inclusions in a Banach space with a noncompact semigroup. Nonlinear Anal. 70, 3424-3436 (2009)

27. Grimmer, R, Pritchard, AJ: Analytic resolvent operators for integral equations in a Banach space. J. Differ. Equ. 50 234-259 (1983)

28. Grimmer, R: Resolvent operators for integral equations in a Banach space. Trans. Am. Math. Soc. 273, 333-349 (1982)

29. Oka, H: Integrated resolvent operators. J. Integral Equ. Appl. 7, 193-232 (1995)

30. Hale, JK, Kato, J: Phase spaces for retarded equations with infinite delay. Funkc. Ekvacioj 21, 11-41 (1978)

31. Yan, Z, Zhang, H: Existence of solutions to impulsive fractional partial neutral stochastic integro-differential inclusions with state-dependent delay. Electron. J. Differ. Equ. 2013, 81 (2013)

32. Pazy, A: Semigroups of Linear Operators and Applications to Partial Differential Equations. Springer, New York (1983)

33. Banas, J, Goebel, K: Measure of Noncompactness in Banach Space. Lecture Notes in Pure and Applied Mathematics. Dekker, New York (1980)

34. Rogovchenko, YV: Nonlinear impulse evolution systems and applications to population models. J. Math. Anal. Appl. 207, 300-315 (1997)

35. Agarwal, R, Meehan, M, O'Regan, D: Fixed Point Theory and Applications. Cambridge Tracts in Mathematics, pp. 178-179. Cambridge University Press, New York (2001)

36. Hino, Y, Murakami, S, Naito, T: Functional-Differential Equations with Infinite Delay. Lecture Notes in Mathematics, vol. 1473. Springer, Berlin (1991) 